\title{
Multidimensional Matrix Inversions and Elliptic Hypergeometric Series on Root Systems
}

\author{
Hjalmar ROSENGREN ${ }^{\dagger}$ and Michael J. SCHLOSSER $\ddagger$ \\ $\dagger$ Department of Mathematics, Chalmers University of Technology \\ and the University of Gothenburg, SE-412 96 Göteborg, Sweden \\ E-mail: hjalmar@chalmers.se \\ URL: http://www.math.chalmers.se/ hjalmar/ \\ $¥$ Fakultät für Mathematik der Universität Wien, \\ Oskar Morgenstern-Platz 1, A-1090 Wien, Austria \\ E-mail: michael.schlosser@univie.ac.at \\ URL: http://www.mat.univie.ac.at/ schlosse/
}

Received May 06, 2020, in final form August 28, 2020; Published online September 24, 2020 https://doi.org/10.3842/SIGMA.2020.088

\begin{abstract}
Multidimensional matrix inversions provide a powerful tool for studying multiple hypergeometric series. In order to extend this technique to elliptic hypergeometric series, we present three new multidimensional matrix inversions. As applications, we obtain a new $A_{r}$ elliptic Jackson summation, as well as several quadratic, cubic and quartic summation formulas.
\end{abstract}

Key words: elliptic hypergeometric series; hypergeometric series associated with root systems; multidimensional matrix inversion

2020 Mathematics Subject Classification: 33D67

\section{Introduction}

Explicit matrix inversions provide a powerful tool in the study of special functions. In particular, they have been used to derive quadratic, cubic and quartic summations for one-variable basic hypergeometric series, see, e.g., [18, 19, 20, 34, 35]. Moreover, Andrews [1] found that the Bailey transform, which is useful for deriving Rogers-Ramanujan-type identities, is closely related to a certain matrix inversion. A unified generalization of several inversions used in these contexts was obtained by Krattenthaler [23]. He proved that the lower-triangular matrices $\left(f_{n k}\right)_{n, k \in \mathbb{Z}}$ and $\left(g_{k l}\right)_{k, l \in \mathbb{Z}}$ with entries

$$
f_{n k}=\frac{\prod_{j=k}^{n-1}\left(a_{j}-c_{k}\right)}{\prod_{j=k+1}^{n}\left(c_{j}-c_{k}\right)}, \quad g_{k l}=\frac{\left(a_{l}-c_{l}\right) \prod_{j=l+1}^{k}\left(a_{j}-c_{k}\right)}{\left(a_{k}-c_{k}\right) \prod_{j=l}^{k-1}\left(c_{j}-c_{k}\right)}
$$

are mutually inverse. The goal of the present work is to obtain generalizations of (1.1) that are both multi-dimensional and elliptic, so that we can apply them to multiple elliptic hypergeometric series.

This paper is a contribution to the Special Issue on Elliptic Integrable Systems, Special Functions and Quantum Field Theory. The full collection is available at https://www.emis.de/journals/SIGMA/elliptic-integrablesystems.html 
Although elliptic hypergeometric series first appeared in the 1980s [13], they did not take off as a mathematical research area until after the seminal paper of Frenkel and Turaev in 1997 [15]. In one of the first papers on this subject, Warnaar gave an elliptic extension of Krattenthaler's matrix inverse [54, Lemma 3.2] and applied it to obtain elliptic analogues of many identities from [18, 19, 20, 35].

Matrix inversion techniques have also been applied to multiple hypergeometric series. One then needs multi-dimensional matrices, which in the cases of relevance to us have rows and columns labelled by $\mathbb{Z}^{r}$. In the 1990's, Milne and coworkers found several multi-dimensional Bailey transforms and matrix inversions, which were applied to basic hypergeometric series associated with root systems [4, 28, 30, 31, 32]. The second author [46, Theorem 3.1] gave a unified extension of these inversions, which reduces to (1.1) in the one-variable case. This allowed him to obtain quadratic and cubic summation formulas for multiple hypergeometric series; see [24, 25, 26, 47, 48, 49, 50, 51] for subsequent work in this direction and [52] for a general overview of classical and basic hypergeometric series associated with root systems.

There has been a substantial amount of work on elliptic hypergeometric series associated with root systems, see [45] and references given there. In particular, Bhatnagar and the second author [5] obtained several elliptic Bailey transforms associated with root systems, and also gave the corresponding multidimensional matrix inversions explicitly. However, until now these have not been extended to the level of generality of (1.1). In the present paper we address this question.

In Section 3 we give two multidimensional extensions of Warnaar's matrix inversion, as well as one somewhat less general inversion. In Section 4 we give applications to summation formulas for elliptic hypergeometric series on root systems. More precisely, in Section 4.2 we obtain a new extension of Jackson's summation and two quadratic summations, in all three cases for series associated with the root system $A_{r}$. In Section 4.3 we obtain a quadratic, a cubic and a quartic summation for series traditionally associated with the root system $D_{r}$. For all these summations except the last one, we also give companion identities, where the summation is over a simplex rather than a hyper-rectangle. One of the quadratic $A_{r}$ summations (Theorem 4.3) and the quartic $D_{r}$ summation (Theorem 4.13) are new even in the limit case of basic hypergeometric series.

The multiple hypergeometric series appearing in this paper are of what different authors have called "type I", "Dixon-type" or "Gustafson-Milne-type". For so called "type II" or "Selberg-type" series, a matrix inversion was found independently by Coskun and Gustafson [12, equation (4.16)] and Rains [36, Corollary 4.3], see also [11] for the corresponding Bailey transform. It is not clear whether that inversion can be extended to the generality of (1.1). A quadratic summation for Selberg-type series is given in [38, Corollary 4.3] and further results are forthcoming [27]. There are also related integral identities [9, 37, 38, 39]. To our knowledge, the present paper contains the first known quadratic summations for multiple elliptic hypergeometric series of type I, and the first known cubic and quartic summations for either type.

There is a close correspondence between elliptic hypergeometric functions given by finite sums and by integrals [45]. In particular, Spiridonov and Warnaar [53] gave several multiple integral inversions, which can be viewed as continuous analogues of matrix inversions from [5]. These integral inversions and their associated Bailey transforms have found a role in quantum field theory, see, e.g., [6, 7, 8, 16, 17, 33, 55]. This may serve as a motivation to look for integral analogues of some of our results.

Finally, we mention that it seems possible to generalize many of our results to transformation formulas. That will be the subject of future work. 


\section{Preliminaries}

\subsection{Theta functions}

We recall some classical results for theta functions. We will write

$$
\theta(x ; p)=\prod_{j=0}^{\infty}\left(1-p^{j} x\right)\left(1-p^{j+1} / x\right),
$$

where $p$ is a complex number with $|p|<1$. We refer to the case $p=0, \theta(x ; 0)=1-x$, as the trigonometric case. We will also use the shorthand notation

$$
\begin{aligned}
& \theta\left(x_{1}, \ldots, x_{n} ; p\right)=\theta\left(x_{1} ; p\right) \cdots \theta\left(x_{n} ; p\right) \\
& \theta\left(x_{1} y^{ \pm}, \ldots, x_{n} y^{ \pm} ; p\right)=\theta\left(x_{1} y ; p\right) \theta\left(x_{1} / y ; p\right) \cdots \theta\left(x_{n} y ; p\right) \theta\left(x_{n} / y ; p\right) .
\end{aligned}
$$

In Section 3.1, we will suppress the dependence on $p$ and simply write $\theta(x)=\theta(x ; p)$.

Among the properties of $\theta$ we mention the inversion formula

$$
\theta(1 / x ; p)=-\frac{1}{x} \theta(x ; p),
$$

the quasi-periodicity

$$
\theta(p x ; p)=-\frac{1}{x} \theta(x ; p)
$$

and Weierstrass' addition formula

$$
\theta(x y, x / y, u v, u / v ; p)-\theta(x v, x / v, u y, u / y ; p)=\frac{u}{y} \theta(y v, y / v, x u, x / u ; p) .
$$

We will also need the identity

$$
\sum_{l=1}^{k} \frac{a_{l} \prod_{j=1}^{k-2} \theta\left(a_{l} b_{j}^{ \pm} ; p\right)}{\prod_{j=1, j \neq l}^{k} \theta\left(a_{l} a_{j}^{ \pm} ; p\right)}=0, \quad k \geq 2
$$

To our knowledge, it was first obtained by Gustafson [21, Lemma 4.14], see [40] for further references and comments.

We will use the following terminology.

Definition 2.1. For $k \in \mathbb{Z}_{\geq 0}, 0<|p|<1$ and $t \in \mathbb{C} \backslash 0$, a theta function of degree $k$, nome $p$ and norm $t$ is a holomorphic function on $\mathbb{C} \backslash\{0\}$ such that

$$
f(p z)=\frac{(-1)^{k} t}{z^{k}} f(z), \quad z \in \mathbb{C} \backslash 0 .
$$

Note that $t$ is not a norm in the sense of normed vector spaces. It is a classical fact (see, e.g., [43, Corollary 1.3.5]) that any function satisfying these conditions can be written as

$$
f(z)=C \theta\left(z / a_{1}, \ldots, z / a_{k} ; p\right), \quad a_{1} \cdots a_{k}=t .
$$

In particular, the zero set of $f$ is the union of the geometric progressions $a_{j} p^{\mathbb{Z}}$. One consequence is the following classical result.

Lemma 2.2. Let $f$ be a theta function of degree $k$, nome $p$ and norm $t$ and assume that

$$
f\left(b_{1}\right)=\cdots=f\left(b_{k}\right)=0,
$$

where $b_{j}$ are non-zero complex numbers such that

$$
b_{j} / b_{k} \notin p^{\mathbb{Z}}, \quad j \neq k, \quad b_{1} \cdots b_{k} \notin t p^{\mathbb{Z}} .
$$

Then, $f$ is identically zero. 


\subsection{Elliptic hypergeometric series}

A one-variable elliptic hypergeometric series $\sum_{k} a_{k}$ is a formal or convergent series such that $a_{k+1} / a_{k}=f(k)$ for some elliptic function $f$. The standard notation for such series is based on elliptic shifted factorials

$$
(a ; q, p)_{k}=\prod_{j=0}^{k-1} \theta\left(a q^{j} ; p\right), \quad k \in \mathbb{Z}_{\geq 0} .
$$

We will use the condensed notation

$$
\left(a_{1}, \ldots, a_{m} ; q, p\right)_{k}=\left(a_{1} ; q, p\right)_{k} \cdots\left(a_{m} ; q, p\right)_{k} .
$$

Moreover, we will often write $|\mathbf{k}|=\sum_{i} k_{i}$.

Throughout the paper we implicitly assume that all parameters are generic, so that we never divide by zero. In particular, it is convenient to assume that $q^{k} \notin p^{\mathbb{Z}}$ for $k \in \mathbb{Z}_{>0}$. Otherwise the factor $(q ; q, p)_{k}$, which frequently appears in denominators, vanishes.

We will write

$$
\Delta(\mathbf{x} ; p)=\Delta\left(x_{1}, \ldots, x_{r} ; p\right)=\prod_{1 \leq i<j \leq r} x_{j} \theta\left(x_{i} / x_{j} ; p\right) .
$$

Then, $\Delta(\mathbf{x} ; 0)=\prod_{1 \leq i<j \leq r}\left(x_{j}-x_{i}\right)$ can be identified with the Weyl denominator of the root system $A_{r-1}$. Similarly, $\Delta(\mathbf{x} ; p)$ is essentially the Weyl denominator of the affine root system $A_{r-1}^{(1)}[29]$.

We will need the $A_{r}$ elliptic Jackson summation

$$
\begin{aligned}
\sum_{\substack{0 \leq k_{i} \leq n_{i} \\
i=1, \ldots, r}} & \frac{\Delta\left(\mathbf{x} q^{\mathbf{k}} ; p\right)}{\Delta(\mathbf{x} ; p)} \prod_{i=1}^{r}\left(\frac{\theta\left(a x_{i} q^{|\mathbf{k}|+k_{i}} ; p\right)}{\theta\left(a x_{i} ; p\right)} \frac{\left(a x_{i} ; q, p\right)_{|\mathbf{k}|}\left(d x_{i}, e x_{i} ; q, p\right)_{k_{i}}}{\left(a x_{i} q^{n_{i}+1} ; q, p\right)_{|\mathbf{k}|}\left(a x_{i} q / b, a x_{i} q / c ; q, p\right)_{k_{i}}}\right) \\
& \times \prod_{i, j=1}^{r} \frac{\left(q^{-n_{j}} x_{i} / x_{j} ; q, p\right)_{k_{i}}}{\left(q x_{i} / x_{j} ; q, p\right)_{k_{i}}} \frac{(b, c ; q, p)_{|\mathbf{k}|}}{(a q / d, a q / e ; q, p)_{|\mathbf{k}|}} q^{|\mathbf{k}|} \\
= & \frac{(a q / b d, a q / c d ; q, p)_{|\mathbf{n}|}}{(a q / d, a q / b c d ; q, p)_{|\mathbf{n}|}} \prod_{i=1}^{r} \frac{\left(a x_{i} q, a x_{i} q / b c ; q, p\right)_{n_{i}}}{\left(a x_{i} q / b, a x_{i} q / c ; q, p\right)_{n_{i}}},
\end{aligned}
$$

where $a^{2} q^{|\mathbf{n}|+1}=b c d e$. It is due to the first author [40, Corollary 5.3] in general and to Milne [30] when $p=0$.

If we introduce the parameters $x_{r+1}=a^{-1}$ and $k_{r+1}=-\sum_{i=1}^{r} k_{i}$, then some factors from (2.5) can be combined to

$$
\Delta\left(\mathbf{x} q^{\mathbf{k}} ; p\right) \prod_{i=1}^{r} \theta\left(a x_{i} q^{|\mathbf{k}|+k_{i}} ; p\right)=\left(x_{r+1} q^{k_{r+1}}\right)^{r} \Delta\left(x_{1} q^{k_{1}}, \ldots, x_{r+1} q^{k_{r+1}} ; p\right) .
$$

This is the reason why (2.5) is associated to $A_{r}$ rather than $A_{r-1}$. Rational limit cases of this type of series play a role in the representation theory of the corresponding unitary group $\mathrm{SU}(r+1)[22]$. In the present paper, we use $A_{r}$ to label also $r$-dimensional series that are derived from (2.5), even though the factor $\prod_{i=1}^{r} \theta\left(a x_{i} q^{|\mathbf{k}|+k_{i}} ; p\right)$ is absent, see, e.g., (4.3).

We will also need the $C_{r}$ elliptic Jackson summation

$$
\sum_{\substack{0 \leq k_{i} \leq n_{i} \\ i=1, \ldots, r}} \frac{\Delta\left(\mathbf{x} q^{\mathbf{k}} ; p\right)}{\Delta(\mathbf{x} ; p)} q^{|\mathbf{k}|} \prod_{1 \leq i \leq j \leq r} \frac{\theta\left(a x_{i} x_{j} q^{k_{i}+k_{j}} ; p\right)}{\theta\left(a x_{i} x_{j} ; p\right)} \prod_{i, j=1}^{r} \frac{\left(q^{-n_{j}} x_{i} / x_{j}, a x_{i} x_{j} ; q, p\right)_{k_{i}}}{\left(q x_{i} / x_{j}, a x_{i} x_{j} q^{n_{j}+1} ; q, p\right)_{k_{i}}}
$$




$$
\begin{aligned}
& \times \prod_{i=1}^{r} \frac{\left(b x_{i}, c x_{i}, d x_{i}, e x_{i} ; q, p\right)_{k_{i}}}{\left(a x_{i} q / b, a x_{i} q / c, a x_{i} q / d, a x_{i} q / e ; q, p\right)_{k_{i}}} \\
= & \frac{\prod_{i, j=1}^{r}\left(a x_{i} x_{j} q ; q, p\right)_{n_{i}}}{\prod_{1 \leq i<j \leq r}\left(a x_{i} x_{j} q ; q, p\right)_{n_{i}+n_{j}}} \frac{(a q / b c, a q / b d, a q / c d ; q, p)_{|\mathbf{n}|}}{\prod_{i=1}^{r}\left(a x_{i} q / b, a x_{i} q / c, a x_{i} q / d, a q^{|\mathbf{n}|-n_{i}+1} / b c d x_{i} ; q, p\right)_{n_{i}}},
\end{aligned}
$$

where $a^{2} q^{|\mathbf{n}|+1}=b c d e$. It is due to the first author [40, Corollary 5.3] in general and, independently, to Denis and Gustafson [14] and Milne and Lilly [32] when $p=0$.

\section{Elliptic multidimensional matrix inversions}

\subsection{General form of the matrix inversions}

We will consider matrices $f=\left(f_{\mathbf{n k}}\right)_{\mathbf{n}, \mathbf{k} \in \mathbb{Z}^{r}}$ labelled by pairs of multi-indices. All matrices that appear are lower-triangular in the sense that $f_{\mathbf{n k}}=0$ unless $\mathbf{n} \geq \mathbf{k}$, that is, $n_{i} \geq k_{i}$ for all $i$. Then, $f$ and $g=\left(g_{\mathbf{n k}}\right)_{\mathbf{n}, \mathbf{k} \in \mathbb{Z}^{r}}$ are inverse matrices if

$$
\sum_{\mathbf{l} \leq \mathbf{k} \leq \mathbf{n}} f_{\mathbf{n k}} g_{\mathbf{k l}}=\delta_{\mathbf{n l}}
$$

or, equivalently,

$$
\sum_{\mathbf{l} \leq \mathbf{k} \leq \mathbf{n}} g_{\mathrm{nk}} f_{\mathrm{kl}}=\delta_{\mathbf{n l}}
$$

Our first main result is the following explicit pair of inverse matrices. Since it will be used to obtain results for multiple hypergeometric series associated with the root system $A_{r}$, we refer to it as an elliptic $A_{r}$ matrix inversion. Here, and in the remainder of Section 3.1, we write $\theta(x)=\theta(x ; p)$, where $p$ is viewed as fixed.

Theorem 3.1 (an elliptic $A_{r}$ matrix inversion). Let $(a(t))_{t \in \mathbb{Z}}$ and $\left(c_{j}(k)\right)_{k \in \mathbb{Z}}, 1 \leq j \leq r$, be arbitrary sequences of scalars. Then the lower-triangular matrices

$$
f_{\mathbf{n k}}=\frac{\prod_{t=|\mathbf{k}|}^{|\mathbf{n}|-1}\left\{\theta\left(a(t) c_{1}\left(k_{1}\right) \cdots c_{r}\left(k_{r}\right)\right) \prod_{j=1}^{r} \theta\left(a(t) / c_{j}\left(k_{j}\right)\right)\right\}}{\prod_{i=1}^{r} \prod_{t=k_{i}+1}^{n_{i}}\left\{\theta\left(c_{i}(t) c_{1}\left(k_{1}\right) \cdots c_{r}\left(k_{r}\right)\right) \prod_{j=1}^{r} \theta\left(c_{i}(t) / c_{j}\left(k_{j}\right)\right)\right\}}
$$

and

$$
\begin{aligned}
g_{\mathbf{k} \mathbf{l}}= & \frac{\theta\left(a(|\mathbf{l}|) c_{1}\left(l_{1}\right) \cdots c_{r}\left(l_{r}\right)\right)}{\theta\left(a(|\mathbf{k}|) c_{1}\left(k_{1}\right) \cdots c_{r}\left(k_{r}\right)\right)} \prod_{1 \leq i<j \leq r} \frac{\theta\left(c_{i}\left(l_{i}\right) / c_{j}\left(l_{j}\right)\right)}{\theta\left(c_{i}\left(k_{i}\right) / c_{j}\left(k_{j}\right)\right)} \prod_{j=1}^{r} \frac{c_{j}\left(l_{j}\right)^{j} \theta\left(a(|\mathbf{l}|) / c_{j}\left(l_{j}\right)\right)}{c_{j}\left(k_{j}\right)^{j} \theta\left(a(|\mathbf{k}|) / c_{j}\left(k_{j}\right)\right)} \\
& \times \frac{\prod_{t=|\mathbf{1}|+1}^{|\mathbf{k}|}\left\{\theta\left(a(t) c_{1}\left(k_{1}\right) \cdots c_{r}\left(k_{r}\right)\right) \prod_{j=1}^{r} \theta\left(a(t) / c_{j}\left(k_{j}\right)\right)\right\}}{\prod_{i=1}^{r} \prod_{t=l_{i}}^{k_{i}-1}\left\{\theta\left(c_{i}(t) c_{1}\left(k_{1}\right) \cdots c_{r}\left(k_{r}\right)\right) \prod_{j=1}^{r} \theta\left(c_{i}(t) / c_{j}\left(k_{j}\right)\right)\right\}}
\end{aligned}
$$

are mutually inverse. 
As we explain in more detail in Section 3.2, Theorem 3.1 contains two matrix inversions due to Bhatnagar and the second author [5] as special cases. The case $p=0$ of Theorem 3.1 is [46, Theorem 3.1]. (The additional parameter $b$ present in [46] may be suppressed by a change of variables.) It contains several previously known matrix inversions [4, 28, 30, 31] as special cases. The case $r=1$ is due to Warnaar [54, Lemma 3.2], see also [44].

We will prove Theorem 3.1 by verifying (3.1). We assume $\mathbf{n}>\mathbf{1}$, since otherwise (3.1) is trivial. Pulling out the factors independent of $\mathbf{k}$, we then have to prove that

$$
\begin{aligned}
& \sum_{\mathbf{1} \leq \mathbf{k} \leq \mathbf{n}} \frac{\prod_{t=|\mathbf{1}|+1}^{|\mathbf{n}|-1}\left\{\theta\left(a(t) c_{1}\left(k_{1}\right) \cdots c_{r}\left(k_{r}\right)\right) \prod_{j=1}^{r} \theta\left(a(t) / c_{j}\left(k_{j}\right)\right)\right\}}{\prod_{i=1}^{r} \prod_{t=l_{i}, t \neq k_{i}}^{n_{i}}\left\{\theta\left(c_{i}(t) c_{1}\left(k_{1}\right) \cdots c_{r}\left(k_{r}\right)\right) \prod_{j=1}^{r} \theta\left(c_{i}(t) / c_{j}\left(k_{j}\right)\right)\right\}} \\
& \quad \times \prod_{1 \leq i<j \leq r} \frac{1}{\theta\left(c_{i}\left(k_{i}\right) / c_{j}\left(k_{j}\right)\right)} \prod_{j=1}^{r} \frac{1}{c_{j}\left(k_{j}\right)^{j}}=0 .
\end{aligned}
$$

After the substitutions $n_{i} \mapsto n_{i}+l_{i}, k_{i} \mapsto k_{i}+l_{i}, c_{i}(k) \mapsto c_{i}\left(k-l_{i}\right)$ (for $\left.i=1, \ldots, r\right)$ and $a(k) \mapsto a(k-|\mathbf{l}|)$, this identity is reduced to the case $\mathbf{l}=\mathbf{0}$. Thus, Theorem 3.1 will follow from the following Lemma.

Lemma 3.2. Let $n_{1}, \ldots, n_{r}$ be non-negative integers, not all equal to zero, and let $a(1), \ldots$, $a(|\mathbf{n}|-1)$ and $c_{j}(k), 1 \leq j \leq r, 0 \leq k \leq n_{j}$, be arbitrary scalars. Then

$$
\begin{aligned}
\sum_{k_{1}, \ldots, k_{r}=0}^{n_{1}, \ldots, n_{r}} \frac{\prod_{t=1}^{|\mathbf{n}|-1}\left\{\theta\left(a(t) c_{1}\left(k_{1}\right) \cdots c_{r}\left(k_{r}\right)\right) \prod_{j=1}^{r} \theta\left(a(t) / c_{j}\left(k_{j}\right)\right)\right\}}{\prod_{i=1}^{r} \prod_{t=0, t \neq k_{i}}^{n_{i}}\left\{\theta\left(c_{i}(t) c_{1}\left(k_{1}\right) \cdots c_{r}\left(k_{r}\right)\right) \prod_{j=1}^{r} \theta\left(c_{i}(t) / c_{j}\left(k_{j}\right)\right)\right\}} \\
\quad \times \prod_{1 \leq i<j \leq r} \frac{1}{\theta\left(c_{i}\left(k_{i}\right) / c_{j}\left(k_{j}\right)\right)} \prod_{j=1}^{r} \frac{1}{c_{j}\left(k_{j}\right)^{j}}=0 .
\end{aligned}
$$

Proof. The case $|\mathbf{n}|=1$ is easily verified. We thus assume $|\mathbf{n}|>1$, and prove the result by induction on $|\mathbf{n}|$. Let $f$ be the left-hand side of the identity, considered as a function of $a(1)$. We observe that $f$ is a theta function of degree $r+1$, nome $p$ and norm 1 . Moreover, if $a(1)=c_{l}(t)$, for some $1 \leq l \leq r$ and $0 \leq t \leq n_{l}$, the term with $k_{l}=t$ vanishes. The sum is then reduced to a sum of the same form, but with $n_{l}$ replaced by $n_{l}-1$, and with $a(1)$ and $c_{l}(t)$ deleted from the parameter sequences. By the induction hypothesis, that sum vanishes. This shows that $f$ has $r+|\mathbf{n}|$ zeroes, which may without loss of generality be considered generic. It follows from Lemma 2.2 that $f$ is identically zero.

Remark 3.3. In the proof we have implicitly assumed $p \neq 0$. When $p=0$, the argument involving theta functions can be replaced by a polynomial argument. Namely, in that case $f$ is a polynomial of degree $r+1$ with $r+|\mathbf{n}|>r+1$ different zeroes, and thus vanishes identically. The resulting proof is different from the one given in [46].

Next we give a matrix inversion that we associate with the root system $B C_{r}$. It contains a matrix inversion from [5] as a special case, see Section 3.2. 
Theorem 3.4 (an elliptic $B C_{r}$ matrix inversion). Let $(a(t))_{t \in \mathbb{Z}}$ and $\left(c_{j}(k)\right)_{k \in \mathbb{Z}}, 1 \leq j \leq r$, be arbitrary sequences of scalars. Then the lower-triangular matrices

$$
f_{\mathbf{n k}}=\frac{\prod_{j=1}^{r} \prod_{t=|\mathbf{k}|}^{|\mathbf{n}|-1} \theta\left(c_{j}\left(k_{j}\right) a(t)^{ \pm}\right)}{\prod_{i, j=1}^{r} \prod_{t=k_{i}+1}^{n_{i}} \theta\left(c_{j}\left(k_{j}\right) c_{i}(t)^{ \pm}\right)}
$$

and

$$
g_{\mathbf{k} \mathbf{l}}=\prod_{1 \leq i<j \leq r} \frac{\theta\left(c_{j}\left(l_{j}\right) c_{i}\left(l_{i}\right)^{ \pm}\right)}{\theta\left(c_{j}\left(k_{j}\right) c_{i}\left(k_{i}\right)^{ \pm}\right)} \prod_{j=1}^{r} \frac{c_{j}\left(k_{j}\right)^{j} \theta\left(c_{j}\left(l_{j}\right) a(|\mathbf{l}|)^{ \pm}\right)}{c_{j}\left(l_{j}\right)^{j} \theta\left(c_{j}\left(k_{j}\right) a(|\mathbf{k}|)^{ \pm}\right)} \frac{\prod_{j=1}^{r} \prod_{t=|\mathbf{l}|+1}^{|\mathbf{k}|} \theta\left(c_{j}\left(k_{j}\right) a(t)^{ \pm}\right)}{\prod_{i, j=1}^{r} \prod_{t=l_{i}}^{k_{i}-1} \theta\left(c_{j}\left(k_{j}\right) c_{i}(t)^{ \pm}\right)}
$$

are mutually inverse.

Theorem 3.4 can be obtained as a special case of [46, Theorem 3.1], that is, of the trigonometric case of Theorem 3.1. More precisely, let $p=0$ in Theorem 3.1, replace $a(t)$ by $b a(t)$ and $c_{j}(k)$ by $b c_{j}(k)$ and then let $b \rightarrow 0$. This gives the matrix inversion

$$
\begin{aligned}
f_{\mathbf{n k}} & =\frac{\prod_{t=|\mathbf{k}|}^{|\mathbf{n}|-1} \prod_{j=1}^{r}\left(1-a(t) / c_{j}\left(k_{j}\right)\right)}{\prod_{i, j=1}^{r} \prod_{t=k_{i}+1}^{n_{i}}\left(1-c_{i}(t) / c_{j}\left(k_{j}\right)\right)}, \\
g_{\mathbf{k} \mathbf{l}} & =\prod_{1 \leq i<j \leq r} \frac{\left(1-c_{i}\left(l_{i}\right) / c_{j}\left(l_{j}\right)\right)}{\left(1-c_{i}\left(k_{i}\right) / c_{j}\left(k_{j}\right)\right)} \prod_{j=1}^{r} \frac{c_{j}\left(l_{j}\right)^{j}\left(1-a(|\mathbf{l}|) / c_{j}\left(l_{j}\right)\right)}{c_{j}\left(k_{j}\right)^{j}\left(1-a(|\mathbf{k}|) / c_{j}\left(k_{j}\right)\right)} \frac{\prod_{t=|\mathbf{l}|+1}^{|\mathbf{k}|} \prod_{j=1}^{r}\left(1-a(t) / c_{j}\left(k_{j}\right)\right)}{\prod_{i, j=1}^{r} \prod_{t=l_{i}}^{k_{i}-1}\left(1-c_{i}(t) / c_{j}\left(k_{j}\right)\right)} .
\end{aligned}
$$

Let us now reintroduce the elliptic nome by substituting

$$
c_{j}(k) \mapsto \frac{\theta\left(c_{j}(k) u^{ \pm}\right)}{\theta\left(c_{j}(k) v^{ \pm}\right)}, \quad a(t) \mapsto \frac{\theta\left(a(t) u^{ \pm}\right)}{\theta\left(a(t) v^{ \pm}\right)} .
$$

After applying (2.3) to all factors, the auxiliary variables $u$ and $v$ only appear in factors that can be cancelled from (3.1). This eventually leads to Theorem 3.4.

It is also possible to prove Theorem 3.4 directly in a similar way as Theorem 3.1. The analogue of Lemma 3.2 is then the identity

$$
\sum_{k_{1}, \ldots, k_{r}=0}^{n_{1}, \ldots, n_{r}} \frac{\prod_{j=1}^{r}\left\{c_{j}\left(k_{j}\right)^{j} \prod_{t=1}^{|\mathbf{n}|-1} \theta\left(c_{j}\left(k_{j}\right) a(t)^{ \pm}\right)\right\}}{\prod_{i, j=1}^{r} \prod_{t=0, t \neq k_{i}}^{n_{i}} \theta\left(c_{j}\left(k_{j}\right) c_{i}(t)^{ \pm}\right) \prod_{1 \leq i<j \leq r} \theta\left(c_{j}\left(k_{j}\right) c_{i}\left(k_{i}\right)^{ \pm}\right)}=0 .
$$

The special case when $n_{j} \equiv 1$ and $c_{j}(0) c_{j}(1)$ is independent of $j$ is equivalent to [37, Lemma 7.8]. Our proof of Theorem 3.1 is a straight-forward applications of Rains' method for proving that special case.

Finally, we give a matrix inversion that we associate with the root system $C_{r}$. The case $p=0$ of Theorem 3.5 is [46, Theorem 4.1]. 
Theorem 3.5 (an elliptic $C_{r}$ matrix inversion). Let $\left(c_{j}(t)\right)_{t \in \mathbb{Z}}, 1 \leq j \leq r$, and $b$ be arbitrary scalars. Then the lower-triangular matrices

$$
f_{\mathbf{n k}}=\prod_{i=1}^{r} \frac{\prod_{t=k_{i}}^{n_{i}-1}\left\{\theta\left(c_{i}(t) b / c_{1}\left(k_{1}\right) \cdots c_{r}\left(k_{r}\right)\right) \prod_{j=1}^{r} \theta\left(c_{i}(t) c_{j}\left(k_{j}\right)\right)\right\}}{\prod_{t=k_{i}+1}^{n_{i}}\left\{\theta\left(c_{i}(t) c_{1}\left(k_{1}\right) \cdots c_{r}\left(k_{r}\right) / b\right) \prod_{j=1}^{r} \theta\left(c_{i}(t) / c_{j}\left(k_{j}\right)\right)\right\}}
$$

and

$$
\begin{aligned}
g_{\mathbf{k} \mathbf{l}}= & \prod_{1 \leq i<j \leq r} \frac{\theta\left(c_{j}\left(l_{j}\right) c_{i}\left(l_{i}\right)^{ \pm}\right)}{\theta\left(c_{j}\left(k_{j}\right) c_{i}\left(k_{i}\right)^{ \pm}\right)} \prod_{j=1}^{r} \frac{c_{j}\left(l_{j}\right)^{r+1-j} \theta\left(c_{j}\left(l_{j}\right)^{2}\right)}{c_{j}\left(k_{j}\right)^{r+1-j} \theta\left(c_{j}\left(k_{j}\right)^{2}\right)} \\
& \times \prod_{i=1}^{r} \frac{\prod_{t=l_{i}+1}^{k_{i}}\left\{\theta\left(c_{i}(t) b / c_{1}\left(k_{1}\right) \cdots c_{r}\left(k_{r}\right)\right) \prod_{j=1}^{r} \theta\left(c_{i}(t) c_{j}\left(k_{j}\right)\right)\right\}}{\prod_{t=l_{i}}^{k_{i}-1}\left\{\theta\left(c_{i}(t) c_{1}\left(k_{1}\right) \cdots c_{r}\left(k_{r}\right) / b\right) \prod_{j=1}^{r} \theta\left(c_{i}(t) / c_{j}\left(k_{j}\right)\right)\right\}}
\end{aligned}
$$

are mutually inverse.

Note that the one-dimensional case of Theorem 3.5 is less general than for Theorems 3.1 and 3.4. Namely, it corresponds to the special case when the sequences $a(t)$ and $c_{1}(t)$ are proportional. We have not been able to extend Theorem 3.5 to the level of generality of the other two inversions. Unfortunately, this means that our method of proof of those results does not apply to Theorem 3.5. Instead we give another proof which is more tedious in its details. The same method can be used to give alternative proofs of Theorems 3.1 and 3.4.

As before, to prove Theorem 3.5 it is enough to verify (3.1) for $\mathbf{n}>\mathbf{l}=\mathbf{0}$, which we state as a Lemma.

Lemma 3.6. Let $n_{1}, \ldots, n_{r}$ be non-negative integers, not all equal to zero, and let $b$ and $c_{j}(k)$, $1 \leq j \leq r, 0 \leq k \leq n_{j}$, be arbitrary scalars. Then

$$
\begin{aligned}
\sum_{k_{1}, \ldots, k_{r}=0}^{n_{1}, \ldots, n_{r}} & \prod_{1 \leq i<j \leq r} \frac{\theta\left(c_{j}\left(k_{j}\right) c_{i}\left(k_{i}\right)\right)}{\theta\left(c_{j}\left(k_{j}\right) / c_{i}\left(k_{i}\right)\right)} \prod_{j=1}^{r} \frac{\theta\left(c_{j}\left(k_{j}\right) b / c_{1}\left(k_{1}\right) \cdots c_{r}\left(k_{r}\right)\right)}{c_{j}\left(k_{j}\right)^{r+1-j}} \\
& \times \prod_{i=1}^{r} \frac{\prod_{t=1}^{n_{i}-1}\left\{\theta\left(c_{i}(t) b / c_{1}\left(k_{1}\right) \cdots c_{r}\left(k_{r}\right)\right) \prod_{j=1}^{r} \theta\left(c_{i}(t) c_{j}\left(k_{j}\right)\right)\right\}}{\prod_{t=0, t \neq k_{i}}^{n_{i}}\left\{\theta\left(c_{i}(t) c_{1}\left(k_{1}\right) \cdots c_{r}\left(k_{r}\right) / b\right) \prod_{j=1}^{r} \theta\left(c_{i}(t) / c_{j}\left(k_{j}\right)\right)\right\}}=0 .
\end{aligned}
$$

Proof. Pulling out all factors independent of $k_{r}$, our sum can be written as

$$
\sum_{k_{1}, \ldots, k_{r-1}=0}^{n_{1}, \ldots, n_{r-1}} A_{k_{1}, \ldots, k_{r-1}} \sum_{k_{r}=0}^{n_{r}} B_{k_{1}, \ldots, k_{r}}
$$

where

$$
\begin{aligned}
A_{k_{1}, \ldots, k_{r-1}}= & \theta\left(b / c_{1}\left(k_{1}\right) \cdots c_{r-1}\left(k_{r-1}\right)\right) \prod_{1 \leq i<j \leq r-1} \frac{\theta\left(c_{j}\left(k_{j}\right) c_{i}\left(k_{i}\right)\right)}{\theta\left(c_{j}\left(k_{j}\right) / c_{i}\left(k_{i}\right)\right)} \\
& \times \prod_{j=1}^{r-1} \frac{\prod_{i=1}^{r} \prod_{t=1}^{n_{i}-1} \theta\left(c_{i}(t) c_{j}\left(k_{j}\right)\right)}{c_{j}\left(k_{j}\right)^{r+1-j} \prod_{i=1}^{r-1} \prod_{t=0, t \neq k_{i}}^{n_{i}} \theta\left(c_{i}(t) / c_{j}\left(k_{j}\right)\right) \prod_{t=0}^{n_{r}} \theta\left(c_{r}(t) / c_{j}\left(k_{j}\right)\right)},
\end{aligned}
$$




$$
\begin{aligned}
B_{k_{1}, \ldots, k_{r}}= & \frac{1}{c_{r}\left(k_{r}\right)} \prod_{j=1}^{r-1} \theta\left(c_{j}\left(k_{j}\right) b / c_{1}\left(k_{1}\right) \cdots c_{r}\left(k_{r}\right), c_{j}\left(k_{j}\right) c_{r}\left(k_{r}\right)\right) \\
& \times \prod_{i=1}^{r} \frac{\prod_{t=1}^{n_{i}-1} \theta\left(c_{i}(t) b / c_{1}\left(k_{1}\right) \cdots c_{r}\left(k_{r}\right), c_{i}(t) c_{r}\left(k_{r}\right)\right)}{\prod_{t=0, t \neq k_{i}}^{n_{i}} \theta\left(c_{i}(t) c_{1}\left(k_{1}\right) \cdots c_{r}\left(k_{r}\right) / b, c_{i}(t) / c_{r}\left(k_{r}\right)\right)} .
\end{aligned}
$$

We now rewrite the inner sum in (3.2) using Gustafson's identity (2.4). Replacing $a_{k} \mapsto$ $a_{k} / \sqrt{\lambda}, b_{k} \mapsto b_{k} \sqrt{\lambda}$ and using (2.1), (2.4) can be written as

$$
\sum_{l=1}^{k} \frac{\prod_{j=1}^{k-2} \theta\left(b_{j} a_{l}, b_{j} \lambda / a_{l}\right)}{\prod_{l=1, j \neq l}^{k} \theta\left(a_{j} a_{l} / \lambda, a_{j} / a_{l}\right)}=0 .
$$

Consider (3.3) with $k=|\mathbf{n}|+1$ and parameters

$$
\begin{aligned}
\left(a_{1}, \ldots, a_{|\mathbf{n}|+1}\right)= & \left(c_{1}(0), \ldots, \widehat{c_{1}\left(k_{1}\right)}, \ldots, c_{1}\left(n_{1}\right), \ldots, c_{r-1}(0), \ldots, \widehat{c_{r-1}\left(k_{r-1}\right.}\right), \ldots \\
& \left.c_{r-1}\left(n_{r-1}\right), c_{r}(0), \ldots, c_{r}\left(n_{r}\right)\right)
\end{aligned}
$$

where the hats signify that the parameter is absent,

$$
\left(b_{1}, \ldots, b_{|\mathbf{n}|-1}\right)=\left(c_{1}(1), \ldots, c_{1}\left(n_{1}-1\right), \ldots, c_{r}(1), \ldots, c_{r}\left(n_{r}-1\right), c_{1}\left(k_{1}\right), \ldots, c_{r-1}\left(k_{r-1}\right)\right)
$$

and $\lambda=b / c_{1}\left(k_{1}\right) \cdots c_{r-1}\left(k_{r-1}\right)$. Then (3.3) splits naturally into $r$ parts, $\sum_{i=1}^{r} S_{i}=0$, where $S_{1}$ is the sum of the first $n_{1}$ terms, $S_{2}$ the following $n_{2}$ terms and so on until the final sum $S_{r}$ which has $n_{r}+1$ terms. It is easy to check that $S_{r}$ equals the inner sum in (3.2), so that

$$
\sum_{k_{r}=0}^{n_{r}} B_{k_{1}, \ldots, k_{r}}=-\sum_{i=1}^{r-1} S_{i}
$$

We will complete the proof by showing that

$$
\sum_{k_{1}, \ldots, k_{r-1}=0}^{n_{1}, \ldots, n_{r-1}} A_{k_{1}, \ldots, k_{r-1}} S_{i}=0, \quad i=1, \ldots, r-1 .
$$

By a symmetry argument, it suffices to do this for $i=1$. We have

$$
S_{1}=\sum_{l=0, l \neq k_{1}}^{n_{1}} C_{l}
$$

with

$$
\begin{aligned}
C_{l}= & \frac{1}{c_{1}(l)} \prod_{j=1}^{r-1} \theta\left(c_{j}\left(k_{j}\right) b / c_{1}(l) c_{1}\left(k_{1}\right) \cdots c_{r-1}\left(k_{r-1}\right), c_{j}\left(k_{j}\right) c_{1}(l)\right) \\
& \times \frac{\prod_{i=1}^{r} \prod_{t=1}^{n_{i}-1} \theta\left(c_{i}(t) b / c_{1}(l) c_{1}\left(k_{1}\right) \cdots c_{r-1}\left(k_{r-1}\right), c_{i}(t) c_{1}(l)\right)}{\prod_{i=1}^{r} \prod_{t=0, t \notin \Lambda_{i}}^{n_{i}} \theta\left(c_{i}(t) c_{1}(l) c_{1}\left(k_{1}\right) \cdots c_{r-1}\left(k_{r-1}\right) / b, c_{i}(t) / c_{1}(l)\right)},
\end{aligned}
$$


where

$$
\Lambda_{i}= \begin{cases}\left\{k_{1}, l\right\}, & i=1, \\ \left\{k_{i}\right\}, & i=2, \ldots, r-1, \\ \emptyset, & i=r .\end{cases}
$$

After some elementary manipulations, we obtain

$$
\begin{aligned}
A_{k_{1}, \ldots, k_{r-1}} C_{l}= & \frac{(-1)^{r} \theta\left(c_{1}\left(k_{1}\right) c_{1}(l), \gamma / c_{1}\left(k_{1}\right), \gamma / c_{1}(l)\right)}{c_{1}\left(k_{1}\right)^{2} c_{1}(l) \theta\left(c_{1}(l) / c_{1}\left(k_{1}\right)\right)} \prod_{2 \leq i<j \leq r-1} \frac{\theta\left(c_{j}\left(k_{j}\right) c_{i}\left(k_{i}\right)\right)}{\theta\left(c_{j}\left(k_{j}\right) / c_{i}\left(k_{i}\right)\right)} \\
& \times \prod_{j=2}^{r-1} \frac{\theta\left(c_{j}\left(k_{j}\right) c_{1}\left(k_{1}\right), c_{j}\left(k_{j}\right) c_{1}(l), c_{j}\left(k_{j}\right) \gamma / c_{1}\left(k_{1}\right) c_{1}(l)\right) \prod_{i=1}^{r} \prod_{t=1}^{n_{i}-1} \theta\left(c_{i}(t) c_{j}\left(k_{j}\right)\right)}{c_{j}\left(k_{j}\right)^{r+2-j} \prod_{t=0}^{n_{1}} \theta\left(c_{1}(t) / c_{j}\left(k_{j}\right)\right) \prod_{i=2}^{r} \prod_{t=0, t \notin \Lambda_{i}}^{n_{i}} \theta\left(c_{i}(t) / c_{j}\left(k_{j}\right)\right)} \\
& \times \prod_{i=1}^{r} \frac{\prod_{t=1}^{n_{i}-1} \theta\left(c_{i}(t) c_{1}\left(k_{1}\right), c_{i}(t) c_{1}(l), c_{i}(t) \gamma / c_{1}\left(k_{1}\right) c_{1}(l)\right)}{\prod_{t=0, t \notin \Lambda_{i}}^{n_{i}} \theta\left(c_{i}(t) / c_{1}\left(k_{1}\right), c_{i}(t) / c_{1}(l), c_{i}(t) c_{1}\left(k_{1}\right) c_{1}(l) / \gamma\right)},
\end{aligned}
$$

where $\gamma=b / c_{2}\left(k_{2}\right) \cdots c_{r}\left(k_{r}\right)$. This is visibly anti-symmetric under interchanging $k_{1} \leftrightarrow l$. Thus,

$$
\sum_{\substack{k_{1}, l=0 \\ k_{1} \neq l}}^{n_{1}} A_{k_{1}, \ldots, k_{r-1}} C_{l}=0
$$

which completes the proof of the theorem.

\subsection{Specializations of the matrix inversions}

For applications to hypergeometric series, one typically specializes the parameters in the matrix inversions to geometric progressions. For ease of reference, we give some specializations of this kind explicitly.

We first consider the special case of Theorem 3.1 when $a(t)=a q^{t}$ and $c_{j}(k)=x_{j} q^{m k}(1 \leq$ $j \leq r$ ), with $m$ a non-zero integer. It will be convenient to let

$$
F_{\mathrm{nk}}=\frac{g_{\mathrm{k} 0} f_{\mathrm{nk}}}{f_{\mathrm{n} 0}}, \quad G_{\mathrm{kl}}=\frac{f_{\mathrm{lo}} g_{\mathrm{kl}}}{g_{\mathrm{k} 0}} .
$$

This is another pair of lower-triangular inverse matrices, which has been normalized so that $F_{\mathbf{n} \mathbf{0}}=G_{\mathbf{n} \mathbf{0}}=1$ for $\mathbf{n} \geq \mathbf{0}$. Let us first assume that $m$ is positive. After some elementary manipulations, the corresponding special case of Theorem 3.1 takes the following form.

Corollary 3.7. When $m$ is a positive integer, the lower-triangular matrices

$$
\begin{aligned}
& F_{\mathbf{n k}}=\frac{\Delta\left(\mathbf{x} q^{m \mathbf{k}} ; p\right)}{\Delta(\mathbf{x} ; p)} \frac{\left(a X q^{|\mathbf{n}|} ; q, p\right)_{m|\mathbf{k}|}}{(a X q ; q, p)_{m|\mathbf{k}|}} q^{m|\mathbf{k}|} \prod_{i, j=1}^{r} \frac{\left(q^{-m n_{j}} x_{i} / x_{j} ; q^{m}, p\right)_{k_{i}}}{\left(q^{m} x_{i} / x_{j} ; q^{m}, p\right)_{k_{i}}} \\
& \times \prod_{i=1}^{r}\left(\frac{\theta\left(X x_{i} q^{m\left(|\mathbf{k}|+k_{i}\right)} ; p\right)}{\theta\left(X x_{i} ; p\right)} \frac{\left(x_{i} / a ; q, p\right)_{m k_{i}}\left(X x_{i} ; q^{m}, p\right)_{|\mathbf{k}|}}{\left(x_{i} q^{1-|\mathbf{n}|} / a ; q, p\right)_{m k_{i}}\left(X x_{i} q^{m\left(n_{i}+1\right)} ; q^{m}, p\right)_{|\mathbf{k}|}}\right)
\end{aligned}
$$


and

$$
\begin{aligned}
& G_{\mathbf{k} \mathbf{l}}=\frac{\Delta\left(\mathbf{x} q^{m \mathbf{l}} ; p\right)}{\Delta(\mathbf{x} ; p)} \frac{\theta\left(a X q^{(m+1)|\mathbf{1}|} ; p\right)}{\theta(a X ; p)} \frac{(a X ; q, p)_{|\mathbf{1}|}}{\left(a X q^{m|\mathbf{k}|+1} ; q, p\right)_{|\mathbf{1}|}} q^{m|\mathbf{1}|} \prod_{i, j=1}^{r} \frac{\left(q^{-m k_{j}} x_{i} / x_{j} ; q^{m}, p\right)_{l_{i}}}{\left(q^{m} x_{i} / x_{j} ; q^{m}, p\right)_{l_{i}}} \\
& \times \prod_{i=1}^{r}\left(\frac{\theta\left(a q^{|\mathbf{1}|-m l_{i}} / x_{i} ; p\right)}{\theta\left(a / x_{i} ; p\right)} \frac{\left(a / x_{i} ; q, p\right)_{|\mathbf{|}|}\left(X x_{i} q^{m|\mathbf{k}|} ; q^{m}, p\right)_{l_{i}}}{\left(a q^{1-m k_{i}} / x_{i} ; q, p\right)_{|\mathbf{1}|}\left(X x_{i} q^{m} ; q^{m}, p\right)_{l_{i}}}\right),
\end{aligned}
$$

where $X=x_{1} \cdots x_{r}$, are mutually inverse.

The case when $m$ is negative can be obtained from Corollary 3.7 simply using the natural interpretation $(a ; q, p)_{-n}=\left(a q^{-n} ; q, p\right)_{n}^{-1}$ for elliptic shifted factorials with negative subscripts. If we replace $m$ by $-m$ and $x_{j}$ by $x_{j}^{-1}$ for $j=1, \ldots, r$, the resulting identity takes the following form.

Corollary 3.8. When $m$ is a positive integer, the lower-triangular matrices

$$
\begin{aligned}
F_{\mathbf{n k}}= & \frac{\Delta\left(\mathbf{x} q^{m \mathbf{k}} ; p\right)}{\Delta(\mathbf{x} ; p)} \frac{(X / a ; q, p)_{m|\mathbf{k}|}}{\left(X q^{1-|\mathbf{n}|} / a ; q, p\right)_{m|\mathbf{k}|}} q^{m|\mathbf{k}|} \prod_{i, j=1}^{r} \frac{\left(q^{-m n_{j}} x_{i} / x_{j} ; q^{m}, p\right)_{k_{i}}}{\left(q^{m} x_{i} / x_{j} ; q^{m}, p\right)_{k_{i}}} \\
& \times \prod_{i=1}^{r}\left(\frac{\theta\left(X x_{i} q^{m\left(|\mathbf{k}|+k_{i}\right)} ; p\right)}{\theta\left(X x_{i} ; p\right)} \frac{\left(a x_{i} q^{|\mathbf{n}|} ; q, p\right)_{m k_{i}}\left(X x_{i} ; q^{m}, p\right)_{|\mathbf{k}|}}{\left(a x_{i} q ; q, p\right)_{m k_{i}}\left(X x_{i} q^{m\left(n_{i}+1\right)} ; q^{m}, p\right)_{|\mathbf{k}|}}\right)
\end{aligned}
$$

and

$$
\begin{aligned}
G_{\mathbf{k} \mathbf{l}}= & \frac{\Delta\left(\mathbf{x} q^{m \mathbf{l}} ; p\right)}{\Delta(\mathbf{x} ; p)} \frac{\theta\left(X q^{(m-1)|\mathbf{1}|} / a ; p\right)}{\theta(X / a ; p)} \frac{(a / X ; q, p)_{|\mathbf{1}|}}{\left(a q^{1-m|\mathbf{k}|} / X ; q, p\right)_{|\mathbf{1}|}} q^{|\mathbf{1}|} \prod_{i, j=1}^{r} \frac{\left(q^{-m k_{j}} x_{i} / x_{j} ; q^{m}, p\right)_{l_{i}}}{\left(q^{m} x_{i} / x_{j} ; q^{m}, p\right)_{l_{i}}} \\
& \times \prod_{i=1}^{r}\left(\frac{\theta\left(a x_{i} q^{|\mathbf{1}|+m l_{i}} ; p\right)}{\theta\left(a x_{i} ; p\right)} \frac{\left(a x_{i} ; q, p\right)_{|\mathbf{|}|}\left(X x_{i} q^{m|\mathbf{k}|} ; q^{m}, p\right)_{l_{i}}}{\left(a x_{i} q^{m k_{i}+1} ; q, p\right)_{|\mathbf{1}|}\left(X x_{i} q^{m} ; q^{m}, p\right)_{l_{i}}}\right),
\end{aligned}
$$

where $X=x_{1} \cdots x_{r}$, are mutually inverse.

Finally, we let $a(t)=a q^{t}$ and $c_{j}(k)=x_{j} q^{m k}(1 \leq j \leq r)$ in Theorem 3.4. Since this result is symmetric under simultaneous inversion of all the parameters $c_{j}(k)$, we may assume that $m$ is positive. This gives the following result.

Corollary 3.9. When $m$ is a positive integer, the lower-triangular matrices

$$
\begin{aligned}
F_{\mathbf{n k}}= & \frac{\Delta\left(\mathbf{x} q^{m \mathbf{k}} ; p\right)}{\Delta(\mathbf{x} ; p)} q^{m|\mathbf{k}|} \prod_{1 \leq i \leq j \leq r} \frac{\theta\left(x_{i} x_{j} q^{m\left(k_{i}+k_{j}\right)} ; p\right)}{\theta\left(x_{i} x_{j} ; p\right)} \\
& \times \prod_{i, j=1}^{r} \frac{\left(q^{-m n_{j}} x_{i} / x_{j}, x_{i} x_{j} ; q^{m}, p\right)_{k_{i}}}{\left(q^{m} x_{i} / x_{j}, q^{m\left(n_{j}+1\right)} x_{i} x_{j} ; q^{m}, p\right)_{k_{i}}} \prod_{i=1}^{r} \frac{\left(a x_{i} q^{|\mathbf{n}|}, x_{i} / a ; q, p\right)_{m k_{i}}}{\left(a x_{i} q, x_{i} q^{1-|\mathbf{n}|} / a ; q, p\right)_{m k_{i}}}
\end{aligned}
$$

and

$$
\begin{aligned}
G_{\mathbf{k} \mathbf{l}}= & \frac{\Delta\left(\mathbf{x} q^{m \mathbf{l}} ; p\right)}{\Delta(\mathbf{x} ; p)} q^{m|\mathbf{1}|} \prod_{1 \leq i<j \leq r} \frac{\theta\left(x_{i} x_{j} q^{m\left(l_{i}+l_{j}\right)} ; p\right)}{\theta\left(x_{i} x_{j} ; p\right)} \prod_{i, j=1}^{r} \frac{\left(q^{-m k_{j}} x_{i} / x_{j}, x_{i} x_{j} q^{m k_{j}} ; q^{m}, p\right)_{l_{i}}}{\left(q^{m} x_{i} / x_{j}, x_{i} x_{j} q^{m} ; q^{m}, p\right)_{l_{i}}} \\
& \times \prod_{i=1}^{r}\left(\frac{\theta\left(a x_{i} q^{|\mathbf{1}|+m l_{i}}, a q^{|\mathbf{1}|-m l_{i}} / x_{i} ; p\right)}{\theta\left(a x_{i}, a / x_{i} ; p\right)} \frac{\left(a x_{i}, a / x_{i} ; q, p\right)_{|\mathbf{1}|}}{\left(a q^{1+m k_{i}} x_{i}, a q^{1-m k_{i}} / x_{i} ; q, p\right)_{|\mathbf{1}|}}\right),
\end{aligned}
$$

are mutually inverse. 
Since Theorem 3.5 lacks the parameters corresponding to $a(t)$ in the other two inversions, we cannot give a specialization at the same level of generality. If we let $c_{j}(k)=x_{j} q^{k}(1 \leq j \leq r)$ we obtain an inversion equivalent to the case $m=1$ of Corollary 3.9, but with $F$ and $G$ interchanged.

One can check that the explicit matrix inversions obtained by Bhatnagar and the second author [5, Corollaries 4.5, 5.8 and 9.6] are equivalent to the case $m=1$ of Corollaries 3.7, 3.8 and 3.9 , respectively.

\section{Applications to elliptic hypergeometric series}

\subsection{Overview}

If $F$ and $G$ are mutually inverse lower-triangular matrices, and there is a known summation formula

$$
\sum_{\mathbf{0} \leq \mathbf{k} \leq \mathbf{n}} F_{\mathbf{n k}} a_{\mathbf{k}}=b_{\mathbf{n}}
$$

then one can immediately deduce the inverse summation

$$
\sum_{\mathbf{0} \leq \mathbf{k} \leq \mathbf{n}} G_{\mathbf{n k}} b_{\mathbf{k}}=a_{\mathbf{n}}
$$

We will obtain several new results by applying this procedure to the multiple Jackson summations (2.5) and (2.6).

In the case of the $A_{r}$ Jackson summation (2.5) this can be done in four ways. Namely, it can be identified (in general, or in some special case) with (4.1), where $F_{\mathbf{n k}}$ is as in Corollary 3.7 or as in Corollary 3.8, in both cases with either $m=1$ or $m=2$. In the case $m=1$ of Corollary 3.8, the identities (4.1) and (4.2) are equivalent. In the other three cases (4.2) gives new summations, see Section 4.2.

The $C_{r}$ Jackson summation (2.6) can be expressed as (4.1), where $F_{\mathbf{n k}}$ is as in Corollary 3.9, with $1 \leq m \leq 4$. The sums (4.2) are then of a form traditionally associated with the root system $D_{r}$ (the motivation for this terminology is weak, but we find it convenient and will use it). The case $m=1$ gives a new proof of the elliptic $D_{r}$ Jackson summation due to the first author [40, Corollary 6.3] (which for $p=0$ is due independently to Bhatnagar [3] and the second author [46]). This is parallel to the proof in [46], and we do not provide the details. The remaining three cases lead to new summations, see Section 4.3.

\subsection{New $A_{r}$ summations}

If we make the change of variables $(a, c) \mapsto\left(X, a X q^{\mid \mathbf{n}} \mid\right)$ in (2.5), it can be expressed as (4.1), where $F_{\mathbf{n k}}$ is as in the case $m=1$ of Corollary 3.7 and

$$
\begin{aligned}
& a_{\mathbf{k}}=\frac{(a X q, b ; q, p)_{|\mathbf{k}|}}{(X q / d, a b d ; q, p)_{\mathbf{k}}} \prod_{i=1}^{r} \frac{\left(d x_{i}, X x_{i} q / a b d ; q, p\right)_{k_{i}}}{\left(x_{i} / a, X x_{i} q / b ; q, p\right)_{k_{i}}}, \\
& b_{\mathbf{k}}=\frac{(X q / b d, a d ; q, p)_{|\mathbf{k}|}}{(X q / d, a b d ; q, p)_{|\mathbf{k}|}} \prod_{i=1}^{r} \frac{\left(X x_{i} q, a b q^{|\mathbf{k}|-k_{i}} / x_{i} ; q, p\right)_{k_{i}}}{\left(X x_{i} q / b, a q^{|\mathbf{k}|-k_{i}} / x_{i} ; q, p\right)_{k_{i}}} .
\end{aligned}
$$

In the inverse identity (4.2), we make the change of variables $x_{i} \mapsto t x_{i}, i=1, \ldots, r, a \mapsto b c d t / a q$, $b \mapsto a q / b c$ and $d \mapsto a q / b d t$, where $t$ is chosen so that after these substitutions $t^{r+1}=a^{2} q / b c d X$. After simplification, we can eliminate $t$ and arrive at the following result. 
Theorem 4.1 (a new $A_{r}$ Jackson summation). We have the summation formula

$$
\begin{gathered}
\sum_{\substack{0 \leq k_{i} \leq n_{i} \\
i=1, \ldots, r}} \frac{\Delta\left(\mathbf{x} q^{\mathbf{k}} ; p\right)}{\Delta(\mathbf{x} ; p)} \frac{\theta\left(a q^{2|\mathbf{k}|} ; p\right)}{\theta(a ; p)} \frac{(a, b, c ; q, p)_{|\mathbf{k}|}}{\left(a q^{|\mathbf{n}|+1}, a q / b, a q / c ; q, p\right)_{|\mathbf{k}|}} q^{|\mathbf{k}|} \prod_{i, j=1}^{r} \frac{\left(q^{-n_{j}} x_{i} / x_{j} ; q, p\right)_{k_{i}}}{\left(q x_{i} / x_{j} ; q, p\right)_{k_{i}}} \\
\quad \times \prod_{i=1}^{r} \frac{\left(b c d / a x_{i} ; q, p\right)_{|\mathbf{k}|-k_{i}}\left(d / x_{i} ; q, p\right)_{|\mathbf{k}|}\left(a^{2} x_{i} q^{|\mathbf{n}|+1} / b c d ; q, p\right)_{k_{i}}}{\left(d / x_{i} ; q, p\right)_{|\mathbf{k}|-k_{i}}\left(b c d q^{-n_{i}} / a x_{i} ; q, p\right)_{|\mathbf{k}|}\left(a x_{i} q / d ; q, p\right)_{k_{i}}} \\
=\frac{(a q, a q / b c ; q, p)_{|\mathbf{n}|}}{(a q / b, a q / c ; q, p)_{|\mathbf{n}|}} \prod_{i=1}^{r} \frac{\left(a x_{i} q / b d, a x_{i} q / c d ; q, p\right)_{n_{i}}}{\left(a x_{i} q / d, a x_{i} q / b c d ; q, p\right)_{n_{i}}}
\end{gathered}
$$

Theorem 4.1 has a companion identity, where the summation is supported on a simplex.

Corollary 4.2. For parameters subject to the relation $a^{2} q^{N+1}=b c_{1} \cdots c_{r+1} d x_{1} \cdots x_{r}$, we have the summation formula

$$
\begin{aligned}
& \sum_{\substack{k_{1}, \ldots, k_{r} \geq 0 \\
0 \leq|\mathbf{k}| \leq N}} \frac{\Delta\left(\mathbf{x} q^{\mathbf{k}} ; p\right)}{\Delta(\mathbf{x} ; p)} \frac{\theta\left(a q^{2|\mathbf{k}|} ; p\right)}{\theta(a ; p)} \frac{\left(a, b, q^{-N} ; q, p\right)_{|\mathbf{k}|}}{\left(a q / b, a q^{N+1} ; q, p\right)_{|\mathbf{k}|}} q^{|\mathbf{k}|} \\
& \quad \times \prod_{i=1}^{r} \frac{\left(a q / C X x_{i} ; q, p\right)_{|\mathbf{k}|-k_{i}}\left(d / x_{i} ; q, p\right)_{|\mathbf{k}|} \prod_{j=1}^{r+1}\left(c_{j} x_{i} ; q, p\right)_{k_{i}}}{\left(d / x_{i} ; q, p\right)_{|\mathbf{k}|-k_{i}}\left(a x_{i} q / d ; q, p\right)_{k_{i}} \prod_{j=1}^{r}\left(q x_{i} / x_{j} ; q, p\right)_{k_{i}}} \frac{1}{i=1} \frac{1}{\left(a c_{i} q / C X ; q, p\right)_{|\mathbf{k}|}} \\
& =\frac{(a q ; q, p)_{N}}{b^{N}(a q / b ; q, p)_{N}} \prod_{i=1}^{r} \frac{\left(a x_{i} q / b d ; q, p\right)_{N}}{\left(a x_{i} q / d ; q, p\right)_{N}} \prod_{i=1}^{r+1} \frac{\left(a q / c_{i} d ; q, p\right)_{N}}{\left(a q / b c_{i} d ; q, p\right)_{N}},
\end{aligned}
$$

where $C=c_{1} \cdots c_{r+1}$ and $X=x_{1} \cdots x_{r}$.

We sketch the standard argument for deducing Corollary 4.2 from Theorem 4.1. One first observes that the case $c_{j}=q^{-n_{j}} / x_{j}$ (for $1 \leq j \leq r$ ) of Corollary 4.2 is equivalent to the case $c=q^{-N}$ of Theorem 4.1. One then uses the quasi-periodicity (2.2) to deduce that Corollary 4.2 holds whenever $c_{j} x_{j} \in p^{\mathbb{Z}} q^{\mathbb{Z} \leq 0}$. The general case then follows by analytic continuation in the parameters $c_{j}$.

The case $p=0$ of Theorem 4.1 and Corollary 4.2 are due to the second author [50, Theorem 4.1, Corollary 4.2]. They generalize multiple ${ }_{6} \phi_{5}$ summations due to Bhatnagar [2]. The general case of Theorem 4.1 has already been announced and applied in several publications. In [41], the first author found that it appears in connection with Felder's SU(2) elliptic quantum group. This led to an explicit system of multivariable biorthogonal functions, which essentially have the summand in (4.3) as their weight function. In [5, Theorem 5.2], Bhatnagar and the second author derived a multiple Bailey transformation ${ }^{1}$ by combining (2.5) and (4.3) (the same result was independently obtained by the first author). Finally, in [42, Theorem 4.1] the first author found another multiple Bailey transformation by combining (4.3) with yet another (Gustafson-Rakha-type) multiple Jackson summation. In fact, we have found a third multiple Bailey transformation by combining (4.3) with itself, but we save that result for future work.

\footnotetext{
${ }^{1}$ This refers to a generalization of Bailey's ${ }_{10} W_{9}$-transformation and should not be confused with the notion of Bailey transform mentioned in the Introduction.
} 
Next, we replace $q$ by $q^{2}$ in (2.5) and then make the substitutions

$$
(a, b, c) \mapsto\left(X, a X q^{|\mathbf{n}|}, a X q^{|\mathbf{n}|+1}\right) .
$$

Using the identity $\left(a, a q ; q^{2}, p\right)_{k}=(a ; q, p)_{2 k}$, we find that (4.1) holds, with $F_{\mathbf{n k}}$ as in the case $m=2$ of Corollary 3.7 and

$$
\begin{aligned}
& a_{\mathbf{k}}=\frac{(a X q ; q, p)_{2|\mathbf{k}|}}{\left(X q^{2} / d, a^{2} d X q ; q^{2}, p\right)_{|\mathbf{k}|}} \prod_{i=1}^{r} \frac{\left(d x_{i}, x_{i} q / a^{2} d ; q^{2}, p\right)_{k_{i}}}{\left(x_{i} / a ; q, p\right)_{2 k_{i}}}, \\
& b_{\mathbf{k}}=q^{-e_{2}(\mathbf{k})} \frac{(a d, q / a d ; q, p)_{|\mathbf{k}|}}{\left(X q^{2} / d, a^{2} d X q ; q^{2}, p\right)_{|\mathbf{k}|}} \prod_{i=1}^{r} \frac{\left(X x_{i} q^{2}, a^{2} X q^{2|\mathbf{k}|-2 k_{i}+1} / x_{i} ; q^{2}, p\right)_{k_{i}}}{\left(a q^{\left.|\mathbf{k}|-k_{i} / x_{i}, x_{i} q^{-|\mathbf{k}|+k_{i}+1} / a ; q, p\right)_{k_{i}}},\right.}
\end{aligned}
$$

where $e_{2}(\mathbf{k})=\sum_{1 \leq i<j \leq r} k_{i} k_{j}$. In the inverse identity (4.2), we make the change of variables $x_{i} \mapsto t x_{i}, i=1, \ldots, r, a \mapsto d t / a q$ and $d \mapsto a b q / d t$, where $t$ is chosen so that after these substitutions $t^{r+1}=a^{2} q / d X$. After simplification, this gives the following result.

Theorem 4.3. If $a^{2} q^{2|\mathbf{n}|+1}=c d$, we have the quadratic summation formula

$$
\begin{aligned}
& \sum_{\substack{0 \leq k_{i} \leq n_{i}, i=1, \ldots, r}} \frac{\Delta\left(\mathbf{x} q^{2 \mathbf{k}} ; p\right)}{\Delta(\mathbf{x} ; p)} \frac{\theta\left(a q^{3|\mathbf{k}|} ; p\right)}{\theta(a ; p)} \frac{(a, b, q / b ; q, p)_{|\mathbf{k}|}}{\left(a q^{2|\mathbf{n}|+1} ; q, p\right)_{|\mathbf{k}|}\left(a q^{2} / b, a q b ; q^{2}, p\right)_{|\mathbf{k}|}} q^{|\mathbf{k}|-e_{2}(\mathbf{k})} \\
& \times \prod_{i=1}^{r} \frac{\left(c x_{i} ; q^{2}, p\right)_{k_{i}}\left(d / x_{i} ; q^{2}, p\right)_{|\mathbf{k}|}\left(d / a x_{i} ; q, p\right)_{|\mathbf{k}|-k_{i}}}{\left(a q^{2|\mathbf{n}|-2 n_{i}+1} / c x_{i} ; q, p\right)_{|\mathbf{k}|}\left(d / x_{i} ; q^{2}, p\right)_{|\mathbf{k}|-k_{i}}\left(a x_{i} q^{k_{i}-|\mathbf{k}|+1} / d ; q\right)_{k_{i}}} \\
& \times \prod_{i, j=1}^{r} \frac{\left(q^{-2 n_{j}} x_{i} / x_{j} ; q^{2}, p\right)_{k_{i}}}{\left(q^{2} x_{i} / x_{j} ; q^{2}, p\right)_{k_{i}}}=\frac{(a q ; q, p)_{2|\mathbf{n}|}}{\left(a b q, a q^{2} / b ; q^{2}, p\right)} \prod_{|\mathbf{n}|}^{r} \frac{\left(a b x_{i} q / d, a x_{i} q^{2} / b d ; q^{2}, p\right)_{n_{i}}}{\left(a x_{i} q / d ; q, p\right)_{2 n_{i}}} .
\end{aligned}
$$

By a standard argument (see the discussion of Corollary 4.2 above) we deduce the following companion identity.

Corollary 4.4. If $a^{2} q=b_{1} \cdots b_{r+1} c x_{1} \cdots x_{r}$, we have the quadratic summation formula

$$
\begin{aligned}
& \sum_{\substack{k_{1}, \ldots, k_{r} \geq 0 \\
|\mathbf{k}| \leq N}} \frac{\Delta\left(\mathbf{x} q^{2 \mathbf{k}} ; p\right)}{\Delta(\mathbf{x} ; p)} \frac{\theta\left(a q^{3|\mathbf{k}|} ; p\right)}{\theta(a ; p)} \frac{\left(a, q^{-N}, q^{N+1} ; q, p\right)_{|\mathbf{k}|}}{\left(a q^{N+2}, a q^{1-N} ; q^{2}, p\right)_{|\mathbf{k}|} \prod_{i=1}^{r+1}\left(a b_{i} q / B X ; q, p\right)_{|\mathbf{k}|}} q^{|\mathbf{k}|-e_{2}(\mathbf{k})} \\
& \times \prod_{i=1}^{r} \frac{\left(c / x_{i} ; q^{2}, p\right)_{|\mathbf{k}|}\left(c / a x_{i} ; q, p\right)_{|\mathbf{k}|-k_{i}} \prod_{j=1}^{r+1}\left(b_{j} x_{i} ; q^{2}, p\right)_{k_{i}}}{\left(c / x_{i} ; q^{2}, p\right)_{|\mathbf{k}|-k_{i}}\left(a x_{i} q^{k_{i}-|\mathbf{k}|+1} / c ; q, p\right)_{k_{i}} \prod_{j=1}^{r}\left(q^{2} x_{i} / x_{j} ; q^{2}, p\right)_{k_{i}}} \\
& = \begin{cases}\frac{\left(a q^{2} ; q^{2}, p\right)_{m}}{\left(q / a ; q^{2}, p\right)_{m}} \prod_{i=1}^{r} \frac{\left(c q / a x_{i} ; q^{2}, p\right)_{m}}{\left(a x_{i} q^{2} / c ; q^{2}, p\right)_{m}} \prod_{i=1}^{r+1} \frac{\left(a q^{2} / b_{i} c ; q^{2}, p\right)_{m}}{\left(b_{i} c q / a ; q^{2}, p\right)_{m}}, & N=2 m, \\
\frac{\left(a q ; q^{2}, p\right)_{m}}{\left(1 / a ; q^{2}, p\right)_{m}} \prod_{i=1}^{r} \frac{\left(c / a x_{i} ; q^{2}, p\right)_{m}}{\left(a x_{i} q / c ; q^{2}, p\right)_{m}} \prod_{i=1}^{r+1} \frac{\left(a q / b_{i} c ; q^{2}, p\right)_{m}}{\left(b_{i} c / a ; q^{2}, p\right)_{m}}, & N=2 m-1,\end{cases}
\end{aligned}
$$

where $B=b_{1} \cdots b_{r+1}$ and $X=x_{1} \cdots x_{r}$. 
Theorem 4.3 and Corollary 4.4 are new even in the case $p=0$. When $r=1$, they reduce to the case $a=b$ of [54, Corollary 4.4] and to [54, Corollary 4.10], respectively. The case when both $p=0$ and $r=1$ is due to Gessel and Stanton [20] and, independently, to Gasper and Rahman [18, 35], respectively.

Finally, we replace $q$ by $q^{2}$ in (2.5) and then make the substitutions

$$
(a, d, e) \mapsto\left(X, a X q^{|\mathbf{n}|}, a X q^{|\mathbf{n}|+1}\right) .
$$

The resulting identity takes the form (4.1), where $F_{\mathbf{n k}}$ is as in the case $m=2$ of Corollary 3.8 and

$$
\begin{aligned}
& a_{\mathbf{k}}=\frac{\left(b, c ; q^{2}, p\right)_{|\mathbf{k}|}}{(X / a ; q, p)_{2|\mathbf{k}|}} \prod_{i=1}^{r} \frac{\left(a x_{i} q ; q, p\right)_{2 k_{i}}}{\left(X x_{i} q^{2} / b, X x_{i} q^{2} / c ; q^{2}, p\right)_{k_{i}}}, \\
& b_{\mathbf{k}}=\frac{(a b / X, X q / a b ; q, p)_{|\mathbf{k}|}}{(a / X, X q / a ; q, p)_{|\mathbf{k}|}} \prod_{i=1}^{r} \frac{\left(X x_{i} q^{2}, X x_{i} q^{2} / b c ; q^{2}, p\right)_{k_{i}}}{\left(X x_{i} q^{2} / b, X x_{i} q^{2} / c ; q^{2}, p\right)_{k_{i}}} .
\end{aligned}
$$

This holds when $a^{2} b c=X^{2} q$. In the inverse identity (4.2), we make the substitutions $x_{i} \mapsto t x_{i}$, $1 \leq i \leq r, a \mapsto a / t, b \mapsto a q b / d, c \mapsto a q^{2} / b d$, where $t$ is chosen so that after these substitutions $t^{r+1}=q a^{2} / c X$. This gives the following quadratic summation formula.

Theorem 4.5. If $a^{2} q^{2|\mathbf{n}|+1}=c d$, then

$$
\begin{aligned}
\sum_{\substack{0 \leq k_{i} \leq n_{i}, i=1, \ldots, r}} & \frac{\Delta\left(\mathbf{x} q^{2 \mathbf{k}} ; p\right)}{\Delta(\mathbf{x} ; p)} \frac{(b, q / b ; q, p)_{|\mathbf{k}|}}{(a q / c, a q / d ; q, p)_{|\mathbf{k}|}} q^{|\mathbf{k}|} \prod_{i, j=1}^{r} \frac{\left(q^{-2 n_{j}} x_{i} / x_{j} ; q^{2}, p\right)_{k_{i}}}{\left(q^{2} x_{i} / x_{j} ; q^{2}, p\right)_{k_{i}}} \\
& \times \prod_{i=1}^{r}\left(\frac{\theta\left(a x_{i} q^{\left.|\mathbf{k}|+2 k_{i} ; p\right)}\right.}{\theta\left(a x_{i} ; p\right)} \frac{\left(a x_{i} ; q, p\right)_{|\mathbf{k}|}}{\left(a x_{i} q^{2 n_{i}+1} ; q, p\right)_{|\mathbf{k}|}} \frac{\left(c x_{i}, d x_{i} ; q^{2}, p\right)_{k_{i}}}{\left(a x_{i} q^{2} / b, a b x_{i} q ; q^{2}, p\right)_{k_{i}}}\right) \\
= & \frac{\left(a q^{2} / b c, a b q / c ; q^{2}, p\right)_{|\mathbf{n}|}}{(a q / c ; q, p)_{2|\mathbf{n}|}} \prod_{i=1}^{r} \frac{\left(a x_{i} q ; q, p\right)_{2 n_{i}}}{\left(a x_{i} q^{2} / b, a b x_{i} q ; q^{2}, p\right)_{n_{i}}} .
\end{aligned}
$$

Theorem 4.5 has the following companion identity.

Corollary 4.6. If $a^{2} q=b_{1} \cdots b_{r+2} x_{1} \cdots x_{r}$, then

$$
\begin{aligned}
& \sum_{\substack{k_{1}, \ldots, k_{r} \geq 0,|\mathbf{k}| \leq N}} \frac{\Delta\left(\mathbf{x} q^{2 \mathbf{k}} ; p\right)}{\Delta(\mathbf{x} ; p)} \frac{\left(q^{-N}, q^{N+1} ; q, p\right)_{|\mathbf{k}|} \mid}{\prod_{i=1}^{r+2}\left(a q / b_{i} ; q, p\right)_{|\mathbf{k}|}} q^{|\mathbf{k}|} \\
& \quad \times \prod_{i=1}^{r}\left(\frac{\theta\left(a x_{i} q^{|\mathbf{k}|+2 k_{i}} ; p\right)}{\theta\left(a x_{i} ; p\right)} \frac{\left(a x_{i} ; q, p\right)_{|\mathbf{k}|} \prod_{j=1}^{r+2}\left(x_{i} b_{j} ; q^{2}, p\right)_{k_{i}}}{\left(a x_{i} q^{N+2}, a x_{i} q^{1-N} ; q^{2}, p\right)_{k_{i}} \prod_{j=1}^{r}\left(q^{2} x_{i} / x_{j} ; q^{2}, p\right)_{k_{i}}}\right) \\
&= \begin{cases}\prod_{i=1}^{r} \frac{\left(a x_{i} q^{2} ; q^{2}, p\right)_{m}}{\left(q / a x_{i} ; q^{2}, p\right)_{m}} \prod_{i=1}^{r+2} \frac{\left(b_{i} q / a ; q^{2}, p\right)_{m}}{\left(a q^{2} / b_{i} ; q^{2}, p\right)_{m}}, \quad N=2 m, \\
\prod_{i=1}^{r} \frac{\left(a x_{i} q ; q^{2}, p\right)_{m}}{\left(1 / a x_{i} ; q^{2}, p\right)_{m}} \prod_{i=1}^{r+2} \frac{\left(b_{i} / a ; q^{2}, p\right)_{m}}{\left(a q / b_{i} ; q^{2}, p\right)_{m}}, & N=2 m-1 .\end{cases}
\end{aligned}
$$

The $p=0$ cases of Theorem 4.5 and Corollary 4.6 are due to the second author [46, Theorems 5.4 and 5.8]. In the case $r=1$, they are equivalent to Theorem 4.3 and hence again reduce to summations from [54]. 


\section{3 $D_{r}$ summations}

A $D_{r}$ quadratic summation formula is obtained if we replace $q$ by $q^{2}$ in (2.6) and then make the substitutions $(a, b, c, d, e) \mapsto\left(1, q / a b, b / a, a q^{|\mathbf{n}|}, a q^{|\mathbf{n}|+1}\right)$. The resulting identity takes the form (4.1), where $F_{\mathbf{n k}}$ is as in the case $m=2$ of Corollary 3.9 and

$$
\begin{aligned}
a_{\mathbf{k}}= & \prod_{i=1}^{r} \frac{\left(a x_{i} q ; q, p\right)_{2 k_{i}}\left(x_{i} q / a b, b x_{i} / a ; q^{2}, p\right)_{k_{i}}}{\left(x_{i} / a ; q, p\right)_{2 k_{i}}\left(a b x_{i} q, a x_{i} q^{2} / b ; q^{2}, p\right)_{k_{i}}}, \\
b_{\mathbf{k}}= & \frac{\left(b q^{1-|\mathbf{k}|}, q^{2-|\mathbf{k}|} / b, a^{2} q ; q^{2}, p\right)_{|\mathbf{k}|} \prod_{i, j=1}^{r}\left(x_{i} x_{j} q^{2} ; q^{2}, p\right)_{k_{i}}}{\prod_{i=1}^{r}\left(a b x_{i} q, a x_{i} q^{2} / b, x_{i} q^{2-|\mathbf{k}|} / a, a q^{1+|\mathbf{k}|-2 k_{i}} / x_{i} ; q^{2}, p\right)_{k_{i}} \prod_{1 \leq i<j \leq r}\left(x_{i} x_{j} q^{2} ; q^{2}, p\right)_{k_{i}+k_{j}}} .
\end{aligned}
$$

The inverse identity (4.2) is easily simplified as follows.

Theorem 4.7. We have the quadratic summation formula

$$
\begin{aligned}
& \sum_{\substack{0 \leq k_{i} \leq n_{i}, i=1, \ldots, r}} \frac{\Delta\left(\mathbf{x} q^{2 \mathbf{k}} ; p\right)}{\Delta(\mathbf{x} ; p)} q^{|\mathbf{k}|-e_{2}(\mathbf{k})} \prod_{1 \leq i<j \leq r} \frac{1}{\left(x_{i} x_{j} ; q^{2}, p\right)_{k_{i}+k_{j}}} \\
& \times \prod_{i=1}^{r}\left(\frac{\theta\left(a x_{i} q^{2 k_{i}+|\mathbf{k}|} ; p\right)}{\theta\left(a x_{i} ; p\right)} \frac{\left(a x_{i} ; q, p\right)_{|\mathbf{k}|}\left(a q / x_{i} ; q, p\right)_{|\mathbf{k}|-k_{i}}}{\left(a x_{i} q^{2 n_{i}+1}, a q^{1-2 n_{i}} / x_{i} ; q, p\right)_{|\mathbf{k}|}\left(q^{k_{i}-|\mathbf{k}|} x_{i} / a ; q, p\right)_{k_{i}}}\right) \\
& \times \frac{\left(a^{2} q ; q^{2}, p\right)_{|\mathbf{k}|}(b, q / b ; q, p)_{|\mathbf{k}|}}{\prod_{i=1}^{r}\left(a b x_{i} q, a x_{i} q^{2} / b ; q^{2}, p\right)_{k_{i}}} \prod_{i, j=1}^{r} \frac{\left(q^{-2 n_{j}} x_{i} / x_{j}, x_{i} x_{j} q^{2 n_{j}} ; q^{2}, p\right)_{k_{i}}}{\left(q^{2} x_{i} / x_{j} ; q^{2}, p\right)_{k_{i}}} \\
& =\prod_{i=1}^{r} \frac{\left(a x_{i} q ; q, p\right)_{2 n_{i}}\left(x_{i} q / a b, b x_{i} / a ; q^{2}, p\right)_{n_{i}}}{\left(x_{i} / a ; q, p\right)_{2 n_{i}}\left(a b x_{i} q, a x_{i} q^{2} / b ; q^{2}, p\right)_{n_{i}}}
\end{aligned}
$$

Theorem 4.7 has two companion identities where the summation is on a simplex. In the first one, we assume that $b=q^{-N}$ and use the standard argument to replace the parameters $q^{-2 n_{j}} / x_{j}$ by generic parameters $b_{j}$.

Corollary 4.8. We have the quadratic summation formula

$$
\begin{aligned}
\sum_{\substack{k_{1}, \ldots, k_{r} \geq 0 \\
|\mathbf{k}| \leq N}} \frac{\Delta\left(\mathbf{x} q^{2 \mathbf{k}} ; p\right)}{\Delta(\mathbf{x} ; p)} q^{|\mathbf{k}|-e_{2}(\mathbf{k})} \frac{\left(a^{2} q ; q^{2}, p\right)_{|\mathbf{k}|}\left(q^{N+1}, q^{-N} ; q, p\right)_{|\mathbf{k}|}}{\prod_{i=1}^{r}\left(a x_{i} q^{N+2}, a x_{i} q^{1-N} ; q^{2}, p\right)_{k_{i}}} \\
\quad \times \prod_{1 \leq i<j \leq r} \frac{1}{\left(x_{i} x_{j} ; q^{2}, p\right)_{k_{i}+k_{j}}} \prod_{i, j=1}^{r} \frac{\left(b_{j} x_{i}, x_{i} / b_{j} ; q^{2}, p\right)_{k_{i}}}{\left(q^{2} x_{i} / x_{j} ; q^{2}, p\right)_{k_{i}}} \\
\quad \times \prod_{i=1}^{r}\left(\frac{\theta\left(a x_{i} q^{2 k_{i}+|\mathbf{k}|} ; p\right)}{\theta\left(a x_{i} ; p\right)} \frac{\left(a x_{i} ; q, p\right)_{|\mathbf{k}|}\left(a q / x_{i} ; q, p\right)_{|\mathbf{k}|-k_{i}}}{\left(a q / b_{i}, a b_{i} q ; q, p\right)_{|\mathbf{k}|}\left(q^{k_{i}-|\mathbf{k}|} x_{i} / a ; q, p\right)_{k_{i}}}\right) \\
= \\
\left\{\begin{array}{l}
\prod_{i=1}^{r} \frac{\left(a x_{i} q^{2}, a q^{2} / x_{i}, b_{i} q / a, q / a b_{i} ; q^{2}, p\right)_{m}}{\left(q / a x_{i}, x_{i} q / a, a q^{2} / b_{i}, a b_{i} q^{2} ; q^{2}, p\right)_{m}}, \quad N=2 m, \\
\prod_{i=1}^{r} \frac{\left(a x_{i} q, a q / x_{i}, b_{i} / a, 1 / a b_{i} ; q^{2}, p\right)_{m}}{\left(1 / a x_{i}, x_{i} / a, a q / b_{i}, a b_{i} q ; q^{2}, p\right)_{m}} \quad N=2 m-1 .
\end{array}\right.
\end{aligned}
$$


In the second companion identity to Theorem 4.7, we first let $a=q^{-N-\frac{1}{2}}$ and then replace $x_{i}$ by $a q^{N+\frac{1}{2}} x_{i}$ for $1 \leq i \leq r$, before applying the standard argument.

Corollary 4.9. We have the quadratic summation formula

$$
\begin{aligned}
\sum_{\substack{k_{1}, \ldots, k_{r} \geq 0,|\mathbf{k}| \leq N}} \frac{\Delta\left(\mathbf{x} q^{2 \mathbf{k}} ; p\right)}{\Delta(\mathbf{x} ; p)} q^{|\mathbf{k}|-e_{2}(\mathbf{k})} \prod_{1 \leq i<j \leq r} \frac{1}{\left(a^{2} x_{i} x_{j} q^{2 N+1} ; q^{2}, p\right)_{k_{i}+k_{j}}} \\
\quad \times \prod_{i=1}^{r}\left(\frac{\theta\left(a x_{i} q^{2 k_{i}+|\mathbf{k}|} ; p\right)}{\theta\left(a x_{i} ; p\right)} \frac{\left(a x_{i} ; q, p\right)_{|\mathbf{k}|}\left(q^{-2 N} / a x_{i} ; q, p\right)_{|\mathbf{k}|-k_{i}}}{\left(a q / c_{i}, c_{i} q^{-2 N} / a ; q, p\right)_{|\mathbf{k}|}\left(q^{2 N+1+k_{i}-|\mathbf{k}|} a x_{i} ; q, p\right)_{k_{i}}}\right) \\
\quad \times \frac{(b, q / b ; q, p)_{|\mathbf{k}|}\left(q^{-2 N} ; q^{2}, p\right)_{|\mathbf{k}|}}{\prod_{i=1}^{r}\left(a b x_{i} q, a x_{i} q^{2} / b ; q^{2}, p\right)_{k_{i}}} \prod_{i, j=1}^{r} \frac{\left(c_{j} x_{i}, a^{2} q^{2 N+1} x_{i} / c_{j} ; q^{2}, p\right)_{k_{i}}}{\left(q^{2} x_{i} / x_{j} ; q^{2}, p\right)_{k_{i}}} \\
=\prod_{i=1}^{r} \frac{\left(a x_{i} q ; q, p\right)_{2 N}\left(a b q / c_{i}, a q^{2} / b c_{i} ; q^{2}, p\right)_{N}}{\left(a / c_{i} ; q, p\right)_{2 N}\left(a x_{i} q^{2} / b, a b x_{i} q ; q^{2}, p\right)_{N}} .
\end{aligned}
$$

The cases $p=0$ of Theorem 4.7, Corollaries 4.8 and 4.9 are due to the second author [46, Theorems 5.21, 5.25 and 5.27]. In the case $r=1$, they are equivalent to Theorem 4.3 and hence again reduce to summations from [54].

A $D_{r}$ cubic summation formula is obtained if we replace $q$ by $q^{3}$ in (2.6) and then make the substitutions $(a, b, c, d, e) \mapsto\left(1, a q^{|\mathbf{n}|}, a q^{|\mathbf{n}|+1}, a q^{|\mathbf{n}|+2}, 1 / a^{3}\right)$. The resulting identity takes the form (4.1), where $F_{\text {nk }}$ is as in the case $m=3$ of Corollary 3.9 and

$$
\begin{aligned}
& a_{\mathbf{k}}=\prod_{i=1}^{r} \frac{\left(a x_{i} q ; q, p\right)_{3 k_{i}}\left(x_{i} / a^{3} ; q^{3}, p\right)_{k_{i}}}{\left(x_{i} / a ; q, p\right)_{3 k_{i}}\left(a^{3} x_{i} q^{3} ; q^{3}, p\right)_{k_{i}}}, \\
& b_{\mathbf{k}}=\frac{\prod_{i, j=1}^{r}\left(x_{i} x_{j} q^{3} ; q^{3}, p\right)_{k_{i}}}{\prod_{1 \leq i<j \leq r}\left(x_{i} x_{j} q^{3} ; q^{3}, p\right)_{k_{i}+k_{j}}} \frac{\left(q^{1-2|\mathbf{k}|} / a^{2}, a^{2} q^{3-|\mathbf{k}|}, a^{2} q^{2-|\mathbf{k}|} ; q^{3}, p\right)_{|\mathbf{k}|}}{\prod_{i=1}^{r}\left(x_{i} q^{1-|\mathbf{k}|} / a, x_{i} q^{3-|\mathbf{k}|} / a, a^{3} x_{i} q^{3}, a q^{1+|\mathbf{k}|-3 k_{i}} / x_{i} ; q^{3}, p\right)_{k_{i}}} .
\end{aligned}
$$

The inverse identity (4.2) is easily simplified as follows.

Theorem 4.10. We have the cubic summation formula

$$
\begin{aligned}
& \sum_{\substack{0 \leq k_{i} \leq n_{i}, i=1, \ldots, r}} \frac{\Delta\left(\mathbf{x} q^{3 \mathbf{k}} ; p\right)}{\Delta(\mathbf{x} ; p)} q^{|\mathbf{k}|-2 e_{2}(\mathbf{k})} \prod_{1 \leq i<j \leq r} \frac{1}{\left(x_{i} x_{j} ; q^{3}, p\right)_{k_{i}+k_{j}}} \\
& \times \prod_{i=1}^{r}\left(\frac{\theta\left(a x_{i} q^{|\mathbf{k}|+3 k_{i}} ; p\right)}{\theta\left(a x_{i} ; p\right)} \frac{\left(a x_{i} ; q, p\right)_{|\mathbf{k}|}\left(a q / x_{i} ; q, p\right)_{|\mathbf{k}|-k_{i}}}{\left(a x_{i} q^{3 n_{i}+1}, a q^{1-3 n_{i}} / x_{i} ; q, p\right)_{|\mathbf{k}|}\left(q^{k_{i}-|\mathbf{k}|} x_{i} / a ; q, p\right)_{2 k_{i}}}\right) \\
& \times \frac{\left(1 / a^{2} ; q, p\right)_{|\mathbf{k}|}\left(a^{2} q ; q, p\right)_{2|\mathbf{k}|}}{\prod_{i=1}^{r}\left(a^{3} x_{i} q^{3} ; q^{3}, p\right)_{k_{i}}} \prod_{i, j=1}^{r} \frac{\left(q^{-3 n_{j}} x_{i} / x_{j}, x_{i} x_{j} q^{3 n_{j}} ; q^{3}, p\right)_{k_{i}}}{\left(q^{3} x_{i} / x_{j} ; q^{3}, p\right)_{k_{i}}} \\
& =\prod_{i=1}^{r} \frac{\left(a x_{i} q ; q, p\right)_{3 n_{i}}\left(x_{i} / a^{3} ; q^{3}, p\right)_{n_{i}}}{\left(x_{i} / a ; q, p\right)_{3 n_{i}}\left(a^{3} x_{i} q^{3} ; q^{3}, p\right)_{n_{i}}} \text {. }
\end{aligned}
$$

We will give two companion identities to Theorem 4.10. For the first one, we first let $a=q^{N / 2}$ and then make the substitutions $x_{i} \mapsto a x_{i} q^{-N / 2}$ for $1 \leq i \leq r$. The standard argument gives the following identity. 
Corollary 4.11. We have the cubic summation formula

$$
\begin{aligned}
\sum_{\substack{k_{1}, \ldots, k_{r} \geq 0 \\
|\mathbf{k}| \leq N}} \frac{\Delta\left(\mathbf{x} q^{3 \mathbf{k}} ; p\right)}{\Delta(\mathbf{x} ; p)} q^{|\mathbf{k}|-2 e_{2}(\mathbf{k})} \prod_{1 \leq i<j \leq r} \frac{1}{\left(a^{2} x_{i} x_{j} q^{-N} ; q^{3}, p\right)_{k_{i}+k_{j}}} \\
\quad \times \prod_{i=1}^{r}\left(\frac{\theta\left(a x_{i} q^{|\mathbf{k}|+3 k_{i}} ; p\right)}{\theta\left(a x_{i} ; p\right)} \frac{\left(a x_{i} ; q, p\right)_{|\mathbf{k}|}\left(q^{N+1} / a x_{i} ; q, p\right)_{|\mathbf{k}|-k_{i}}}{\left(a q / b_{i}, b_{i} q^{N+1} / a ; q, p\right)_{|\mathbf{k}|}\left(q^{k_{i}-|\mathbf{k}|-N} a x_{i} ; q, p\right)_{2 k_{i}}}\right) \\
\\
\quad \times \frac{\left(q^{-N} ; q, p\right)_{|\mathbf{k}|}\left(q^{N+1} ; q, p\right)_{2|\mathbf{k}|}}{\prod_{i, j=1}^{r}} \frac{\left(b_{j} x_{i}, a^{2} x_{i} q^{-N} / b_{j} ; q^{3}, p\right)_{k_{i}}}{\left(q^{3} x_{i} / x_{j} ; q^{3}, p\right)_{k_{i}}} \\
=\prod_{i=1}^{r} \frac{\left.\left(b_{i} / a ; q, p\right)_{N+1}\left(q^{N+3} ; q^{3}, p\right)_{k_{i}} / a x_{i} ; q^{3}, p\right)_{N+1}}{\left(1 / a x_{i} ; q, p\right)_{N+1}\left(b_{i} q^{-N} / a ; q^{3}, p\right)_{N+1}}
\end{aligned}
$$

Next, we let $a=q^{-(N+1) / 2}$ in Theorem 4.10, so that the factor $\left(a^{2} q ; q, p\right)_{2|\mathbf{k}|}$ vanishes unless $2|\mathbf{k}| \leq N$. As before, this gives the following companion identity.

Corollary 4.12. We have the cubic summation formula

$$
\begin{aligned}
\sum_{\substack{k_{1}, \ldots, k_{r} \geq 0 \\
2|\mathbf{k}| \leq N}} \frac{\Delta\left(\mathbf{x} q^{3 \mathbf{k}} ; p\right)}{\Delta(\mathbf{x} ; p)} q^{|\mathbf{k}|-2 e_{2}(\mathbf{k})} \prod_{1 \leq i<j \leq r} \frac{1}{\left(a^{2} x_{i} x_{j} q^{N+1} ; q^{3}, p\right)_{k_{i}+k_{j}}} \\
\quad \times \prod_{i=1}^{r}\left(\frac{\theta\left(a x_{i} q^{|\mathbf{k}|+3 k_{i}} ; p\right)}{\theta\left(a x_{i} ; p\right)} \frac{\left(a x_{i} ; q, p\right)_{|\mathbf{k}|}\left(q^{-N} / a x_{i} ; q, p\right)_{|\mathbf{k}|-k_{i}}}{\left(a q / b_{i}, b_{i} q^{-N} / a ; q, p\right)_{|\mathbf{k}|}\left(q^{k_{i}-|\mathbf{k}|+N+1} a x_{i} ; q, p\right)_{2 k_{i}}}\right) \\
\times \\
\times \frac{\left(q^{N+1} ; q, p\right)_{|\mathbf{k}|}\left(q^{-N} ; q, p\right)_{2|\mathbf{k}|}}{\prod_{i=1}^{r}\left(a x_{i} q^{2-N} ; q^{3}, p\right)_{k_{i}}} \frac{\left(b_{j} x_{i}, a^{2} x_{i} q^{N+1} / b_{j} ; q^{3}, p\right)_{k_{i}}}{\left(q^{3} x_{i} / x_{j} ; q^{3}, p\right)_{k_{i}}} \\
=\prod_{i=1}^{r} \frac{\left(a x_{i} q ; q, p\right)_{N}\left(a q^{2-N} / b_{i} ; q^{3}, p\right)_{N}}{\left(a q / b_{i} ; q, p\right)_{N}\left(a x_{i} q^{2-N} ; q^{3}, p\right)_{N}}
\end{aligned}
$$

The cases $p=0$ of Theorem 4.10, Corollaries 4.11 and 4.12 are due to the second author [46, Theorems 5.29 and 5.34]. The case $r=1$ of Theorem 4.10 is equivalent to the case $b=a$ of [54, Corollary 4.5] and the corollaries to [54, Corollaries 4.14 and 4.12], respectively. The case when both $p=0$ and $r=1$ of all these results are due to Gasper [18].

Finally, to obtain a quartic summation from (2.6), we first replace $q$ by $q^{4}$ and then make the substitutions $(a, b, c, d, e) \mapsto\left(1, a q^{|\mathbf{n}|}, a q^{|\mathbf{n}|+1}, a q^{|\mathbf{n}|+2}, a q^{|\mathbf{n}|+3}\right)$. This is only possible if $a^{4}=q^{-2}$. The case $a=q^{-1 / 2}$ leads to a trivial identity, so we will only consider the case $a=\mathrm{i} q^{-1 / 2}$. We find that (4.1) holds, where $F_{\mathbf{n k}}$ is as in Corollary 3.9 with $m=4$ and $a=\mathrm{i} q^{-1 / 2}$, and

$$
\begin{aligned}
a_{\mathbf{k}} & =\prod_{i=1}^{r} \frac{\left(\mathrm{i} x_{i} q^{\frac{1}{2}} ; q, p\right)_{4 k_{i}}}{\left(-\mathrm{i} x_{i} q^{\frac{1}{2}} ; q, p\right)_{4 k_{i}}} \\
b_{\mathbf{k}} & =\frac{\prod_{i, j=1}^{r}\left(x_{i} x_{j} q^{4} ; q^{4}, p\right)_{k_{i}}}{\prod_{1 \leq i<j \leq r}\left(x_{i} x_{j} q^{4} ; q^{4}, p\right)_{k_{i}+k_{j}}}
\end{aligned}
$$




$$
\begin{aligned}
& \times \frac{\left(-q^{2-2|\mathbf{k}|},-q^{3-2|\mathbf{k}|},-q^{4-2|\mathbf{k}|} ; q^{4}, p\right)_{|\mathbf{k}|}}{\prod_{i=1}^{r}\left(-\mathrm{i} x_{i} q^{\frac{5}{2}-|\mathbf{k}|},-\mathrm{i} x_{i} q^{\frac{7}{2}-|\mathbf{k}|},-\mathrm{i} x_{i} q^{\frac{9}{2}-|\mathbf{k}|}, \mathrm{i} q^{|\mathbf{k}|-4 k_{i}+\frac{5}{2}} / x_{i} ; q^{4}, p\right)_{k_{i}}} \\
= & q^{-3|\mathbf{k}|-3 e_{2}(\mathbf{k})}\left(-1,-q,-q^{2} ; q^{2}, p\right)_{|\mathbf{k}|} \frac{\prod_{1 \leq i<j=1}^{r}\left(x_{i} x_{j} q^{4} ; q^{4}, p\right)_{k_{i}}}{\prod_{1 \leq r}\left(x_{i} x_{j} q^{4} ; q^{4}, p\right)_{k_{i}+k_{j}}} \\
& \times \prod_{i=1}^{r}\left(\frac{\theta\left(\mathrm{i} q^{-\frac{1}{2}} / x_{i} ; p\right)}{\theta\left(\mathrm{i} q^{|\mathbf{k}|-4 k_{i}+\frac{1}{2}} / x_{i} ; p\right)} \frac{\left(\mathrm{i} q^{\frac{1}{2}} / x_{i} ; q, p\right)_{|\mathbf{k}|-k_{i}}}{\left(\mathrm{i} q^{-\frac{1}{2}} / x_{i} ; q, p\right)_{|\mathbf{k}|}\left(-\mathrm{i} x_{i} q^{k_{i}-|\mathbf{k}|+\frac{1}{2}} ; q, p\right)_{3 k_{i}}}\right)
\end{aligned}
$$

where the second expression for $b_{\mathbf{k}}$ is obtained by some routine manipulations. In the corresponding identity (4.2), we reintroduce the parameter $a$ by substituting $x_{i} \mapsto-\mathrm{i} a x_{i} q^{\frac{1}{2}}, 1 \leq i \leq r$. This leads to the following result.

Theorem 4.13. We have the quartic summation formula

$$
\begin{aligned}
& \sum_{\substack{0 \leq k_{i} \leq n_{i}, i=1, \ldots, r}} \frac{\Delta\left(\mathbf{x} q^{4 \mathbf{k}} ; p\right)}{\Delta(\mathbf{x} ; p)} q^{|\mathbf{k}|-3 e_{2}(\mathbf{k})} \frac{\left(-1,-q,-q^{2} ; q^{2}, p\right)_{|\mathbf{k}|}}{\prod_{1 \leq i<j \leq r}\left(-a^{2} x_{i} x_{j} q ; q^{4}, p\right)_{k_{i}+k_{j}}} \\
& \times \prod_{i=1}^{r}\left(\frac{\theta\left(a x_{i} q^{|\mathbf{k}|+4 k_{i}} ; p\right)}{\theta\left(a x_{i} ; p\right)} \frac{\left(a x_{i} ; q, p\right)_{|\mathbf{k}|}\left(-1 / a x_{i} ; q, p\right)_{|\mathbf{k}|-k_{i}}}{\left(a x_{i} q^{4 n_{i}+1},-q^{-4 n_{i}} / a x_{i} ; q, p\right)_{|\mathbf{k}|}\left(-q^{k_{i}-|\mathbf{k}|+1} a x_{i} ; q, p\right)_{3 k_{i}}}\right) \\
& \times \prod_{i, j=1}^{r} \frac{\left(q^{-4 n_{j}} x_{i} / x_{j},-a^{2} x_{i} x_{j} q^{4 n_{j}+1} ; q^{4}, p\right)_{k_{i}}}{\left(q^{4} x_{i} / x_{j} ; q^{4}, p\right)_{k_{i}}}=\prod_{i=1}^{r} \frac{\left(a x_{i} q ; q, p\right)_{4 n_{i}}}{\left(-a x_{i} q ; q, p\right)_{4 n_{i}}} .
\end{aligned}
$$

Theorem 4.13 is a new result even when $p=0$. In fact, we are not aware of any other quartic summations for multiple hypergeometric series. The case $r=1$ (where we have put $x_{1}=1$ without loss of generality) is

$$
\sum_{k=0}^{n} \frac{\theta\left(a q^{5 k} ; p\right)}{\theta(a ; p)} \frac{(a ; q, p)_{k}(-1 ; q, p)_{2 k}\left(q^{-4 n},-a^{2} q^{4 n+1} ; q^{4}, p\right)_{k}}{\left(a q^{4 n+1},-q^{-4 n} / a ; q, p\right)_{k}(-a q ; q, p)_{3 k}\left(q^{2} ; q^{2}, p\right)_{k}} q^{k}=\frac{(a q ; q, p)_{4 n}}{(-a q ; q, p)_{4 n}}
$$

This is the case $b=-a q$ of Warnaar's "less appealing quartic transformation", that is, the penultimate identity in [54]. More explicitly, it is given as [10, Corollary 2.5]. The case $p=0$ of (4.5) is equivalent to the special case $a b=-q^{1-2 n}$ of [18, equation (5.28)].

\section{Acknowledgements}

We thank the anonymous referees for several useful comments. The first author was partially supported by the Swedish Science Research Council. The second author was partially supported by Austrian Science Fund grants S9607 and P32305.

\section{References}

[1] Andrews G.E., Connection coefficient problems and partitions, in Relations between Combinatorics and other Parts of Mathematics (Ohio State University, Columbus, Ohio, 1978), Proc. Sympos. Pure Math., Vol. 34, Amer. Math. Soc., Providence, R.I., 1979, 1-24.

[2] Bhatnagar G., Inverse relations, generalized bibasic series, and the U(n) extensions, Ph.D. Thesis, The Ohio State University, 1995. 
[3] Bhatnagar G., $D_{n}$ basic hypergeometric series, Ramanujan J. 3 (1999), 175-203.

[4] Bhatnagar G., Milne S.C., Generalized bibasic hypergeometric series and their U(n) extensions, Adv. Math. 131 (1997), 188-252.

[5] Bhatnagar G., Schlosser M.J., Elliptic well-poised Bailey transforms and lemmas on root systems, SIGMA 14 (2018), 025, 44 pages, arXiv:1704.00020.

[6] Brünner F., Spiridonov V.P., A duality web of linear quivers, Phys. Lett. B 761 (2016), 261-264, arXiv:1605.06991.

[7] Brünner F., Spiridonov V.P., $4 \mathrm{~d} \mathcal{N}=1$ quiver gauge theories and the $A_{n}$ Bailey lemma, J. High Energy Phys. 2018 (2018), no. 3, 105, 30 pages, arXiv:1712.07018.

[8] Buican M., Laczko Z., Nishinaka T., $\mathcal{N}=2$ S-duality revisited, J. High Energy Phys. 2017 (2017), no. 9, 087, 36 pages, arXiv:1706.03797.

[9] van de Bult F.J., Two multivariate quadratic transformations of elliptic hypergeometric integrals, arXiv:1109.1123.

[10] Chu W., Jia C., Quartic theta hypergeometric series, Ramanujan J. 32 (2013), 23-81.

[11] Coskun H., An elliptic $B C_{n}$ Bailey lemma, multiple Rogers-Ramanujan identities and Euler's pentagonal number theorems, Trans. Amer. Math. Soc. 360 (2008), 5397-5433.

[12] Coskun H., Gustafson R.A., Well-poised Macdonald functions $W_{\lambda}$ and Jackson coefficients $\omega_{\lambda}$ on $B C_{n}$, in Jack, Hall-Littlewood and Macdonald Polynomials, Contemp. Math., Vol. 417, Amer. Math. Soc., Providence, RI, 2006, 127-155, arXiv:math.CO/0412153.

[13] Date E., Jimbo M., Kuniba A., Miwa T., Okado M., Exactly solvable SOS models. II. Proof of the startriangle relation and combinatorial identities, in Conformal Field Theory and Solvable Lattice Models (Kyoto, 1986), Adv. Stud. Pure Math., Vol. 16, Academic Press, Boston, MA, 1988, 17-122.

[14] Denis R.Y., Gustafson R.A., An SU(n) $q$-beta integral transformation and multiple hypergeometric series identities, SIAM J. Math. Anal. 23 (1992), 552-561.

[15] Frenkel I.B., Turaev V.G., Elliptic solutions of the Yang-Baxter equation and modular hypergeometric functions, in The Arnold-Gelfand mathematical seminars, Birkhäuser Boston, Boston, MA, 1997, 171-204.

[16] Gadde A., Rastelli L., Razamat S.S., Yan W., The superconformal index of the E6 SCFT, J. High Energy Phys. 2010 (2010), no. 8, 107, 27 pages, arXiv:1003.4244.

[17] Gaiotto D., Kim H.-C., Duality walls and defects in 5 d $\mathcal{N}=1$ theories, J. High Energy Phys. 2017 (2017), no. 1, 019, 57 pages, arXiv:1506.03871.

[18] Gasper G., Summation, transformation, and expansion formulas for bibasic series, Trans. Amer. Math. Soc. 312 (1989), 257-277.

[19] Gasper G., Rahman M., An indefinite bibasic summation formula and some quadratic, cubic and quartic summation and transformation formulas, Canad. J. Math. 42 (1990), 1-27.

[20] Gessel I., Stanton D., Applications of $q$-Lagrange inversion to basic hypergeometric series, Trans. Amer. Math. Soc. 277 (1983), 173-201.

[21] Gustafson R.A., Multilateral summation theorems for ordinary and basic hypergeometric series in $\mathrm{U}(n)$, SIAM J. Math. Anal. 18 (1987), 1576-1596.

[22] Holman III W.J., Biedenharn L.C., Louck J.D., On hypergeometric series well-poised in SU(n), SIAM J. Math. Anal. 7 (1976), 529-541.

[23] Krattenthaler C., A new matrix inverse, Proc. Amer. Math. Soc. 124 (1996), 47-59.

[24] Krattenthaler C., Schlosser M.J., A new multidimensional matrix inverse with applications to multiple q-series, Discrete Math. 204 (1999), 249-279.

[25] Lassalle M., Schlosser M.J., Inversion of the Pieri formula for Macdonald polynomials, Adv. Math. 202 (2006), 289-325, arXiv:math.CO/0402127.

[26] Lassalle M., Schlosser M.J., Recurrence formulas for Macdonald polynomials of type A, J. Algebraic Combin. 32 (2010), 113-131, arXiv:0902.2099.

[27] Lee C., Rains E.M., Warnaar S.O., An elliptic hypergeometric function approach to branching rules, arXiv:2007.03174.

[28] Lilly G.M., Milne S.C., The $C_{l}$ Bailey transform and Bailey lemma, Constr. Approx. 9 (1993), $473-500$.

[29] Macdonald I.G., Affine root systems and Dedekind's $\eta$-function, Invent. Math. 15 (1972), 91-143. 
[30] Milne S.C., Balanced ${ }_{3} \phi_{2}$ summation theorems for $\mathrm{U}(n)$ basic hypergeometric series, Adv. Math. 131 (1997), 93-187.

[31] Milne S.C., Lilly G.M., The $A_{l}$ and $C_{l}$ Bailey transform and lemma, Bull. Amer. Math. Soc. (N.S.) 26 (1992), 258-263, arXiv:math.CA/9204236.

[32] Milne S.C., Lilly G.M., Consequences of the $A_{l}$ and $C_{l}$ Bailey transform and Bailey lemma, Discrete Math. 139 (1995), 319-346.

[33] Nazzal B., Razamat S.S., Surface defects in E-string compactifications and the van Diejen model, SIGMA 14 (2018), 036, 20 pages, arXiv:1801.00960.

[34] Rahman M., Some cubic summation formulas for basic hypergeometric series, Utilitas Math. 36 (1989), 161-172.

[35] Rahman M., Some quadratic and cubic summation formulas for basic hypergeometric series, Canad. J. Math. 45 (1993), 394-411.

[36] Rains E.M., $B C_{n}$-symmetric Abelian functions, Duke Math. J. $135 \quad$ (2006), 99-180, arXiv:math.CO/0402113.

[37] Rains E.M., Transformations of elliptic hypergeometric integrals, Ann. of Math. 171 (2010), 169-243, arXiv:math.QA/0309252.

[38] Rains E.M., Elliptic Littlewood identities, J. Combin. Theory Ser. A 119 (2012), 1558-1609, arXiv:0806.0871.

[39] Rains E.M., Multivariate quadratic transformations and the interpolation kernel, SIGMA 14 (2018), 019, 69 pages, arXiv:1408.0305.

[40] Rosengren H., Elliptic hypergeometric series on root systems, Adv. Math. 181 (2004), 417-447, arXiv:math.CA/0207046.

[41] Rosengren H., Felder's elliptic quantum group and elliptic hypergeometric series on the root system $A_{n}$, Int. Math. Res. Not. 2011 (2011), 2861-2920, arXiv:1003.3730.

[42] Rosengren H., Gustafson-Rakha-type elliptic hypergeometric series, SIGMA 13 (2017), 037, 11 pages, arXiv:1701.08960.

[43] Rosengren H., Elliptic hypergeometric functions, in Lectures on Orthogonal Polynomials and Special Functions, Cambridge University Press, Cambridge, to appear, arXiv:1608.06161.

[44] Rosengren H., Schlosser M.J., On Warnaar's elliptic matrix inversion and Karlsson-Minton-type elliptic hypergeometric series, J. Comput. Appl. Math. 178 (2005), 377-391, arXiv:math.CA/0309358.

[45] Rosengren H., Warnaar S.O., Elliptic hypergeometric functions associated with root systems, in Encyclopedia of Special Functions: The Askey-Bateman Project, Vol. 2, Multivariable Special Functions, Cambridge University Press, Cambridge, 2020, 159-186, arXiv:1704.08406.

[46] Schlosser M.J., Multidimensional matrix inversions and $A_{r}$ and $D_{r}$ basic hypergeometric series, Ramanujan J. 1 (1997), 243-274.

[47] Schlosser M.J., Some new applications of matrix inversions in $A_{r}$, Ramanujan J. 3 (1999), 405-461.

[48] Schlosser M.J., A new multidimensional matrix inversion in $A_{r}$, in $q$-Series from a Contemporary Perspective (South Hadley, MA, 1998), Contemp. Math., Vol. 254, Amer. Math. Soc., Providence, RI, 2000, $413-432$.

[49] Schlosser M.J., Macdonald polynomials and multivariable basic hypergeometric series, SIGMA 3 (2007), 056, 30 pages, arXiv:math.CO/0611639.

[50] Schlosser M.J., A new multivariable ${ }_{6} \psi_{6}$ summation formula, Ramanujan J. 17 (2008), 305-319, arXiv:math.CA/0607122.

[51] Schlosser M.J., Multilateral inversion of $A_{r}, C_{r}$, and $D_{r}$ basic hypergeometric series, Ann. Comb. 13 (2009), 341-363, arXiv:math.CA/0608742.

[52] Schlosser M.J., Hypergeometric and basic hypergeometric series and integrals associated with root systems, in Encyclopedia of Special Functions: The Askey-Bateman Project, Vol. 2, Multivariable Special Functions, Cambridge University Press, Cambridge, 2020, 122-158, arXiv:1705.09221.

[53] Spiridonov V.P., Warnaar S.O., Inversions of integral operators and elliptic beta integrals on root systems, Adv. Math. 207 (2006), 91-132, arXiv:math.CA/0411044.

[54] Warnaar S.O., Summation and transformation formulas for elliptic hypergeometric series, Constr. Approx. 18 (2002), 479-502, arXiv:math.QA/0001006.

[55] Yagi J., Surface defects and elliptic quantum groups, J. High Energy Phys. 2017 (2017), no. 6, 013, 32 pages, arXiv:1701.05562. 